\title{
STOKES FLOW AROUND A BEND
}

\author{
$\mathrm{BY}$ \\ S. A. KHURI (Department of Computer and Mathematical Sciences, University of \\ Houston-Downtown, Houston, Texas) \\ AND \\ C. Y. WANG (Department of Mathematics, Michigan State University, East Lansing, Michigan)
}

\begin{abstract}
The matched eigenfunction expansion method is used for solving Stokes flow around a channel bend. The flow region is decomposed into rectangular and cylindrical subregions. This enables the stream function to be represented by means of an expansion of Papkovich-Fadle eigenfunctions in each of these two subregions. The coefficients in these expansions are determined by imposing weak $C^{3}$ continuity of the stream function across subregion interfaces and then taking advantage of the biorthogonality conditions in both cylindrical and rectangular coordinates.
\end{abstract}

1. Introduction. The flow and pressure drop through a pipe or channel bend is extremely important in the design of hydraulic systems. Due to the difficulty of theoretical analysis almost all data are experimental. If the size of the bend is small, such as that used in micro-fluids, the Reynolds number is very low and Stokes flow is valid.

In this paper the matched eigenfunction expansion method is used to solve Stokes flow around a channel bend. The use of the matching technique is motivated by the work of Trogdon and Joseph [22] in their study of plane flow of a liquid over a rectangular slot. They split the region into four subregions, which enables the stream function to be represented by means of an expansion of cartesian Papkovich-Fadle eigenfunctions in each subregion. Imposing boundary conditions and continuity of velocities and stresses across the common interfaces yields relations which were inverted using biorthogonality conditions. This led to an infinite system of linear equations which was then solved by truncations.

Phillips [17] used a similar method for solving Stokes flow around a two-dimensional constriction. The flow region is decomposed into a number of rectangular subregions. Within each subregion the stream function is represented by an expansion of eigenfunctions of the biharmonic operator. The coefficients in these expansions are determined by imposing $C^{3}$ continuity of the stream function across subregion interfaces and then taking advantage of the biorthogonality conditions. A post-processing technique is also

Received January 5, 1995.

1991 Mathematics Subject Classification. Primary 76D99, 31B30.

(C)1997 Brown University 
developed to improve the accuracy of the approximation around corners by determining the coefficients in the known locally convergent expansions of the stream function there.

Phillips and Davies (1988) [18] used the method of matched eigenfunction expansions to solve Poisson's equation in a contraction region. They also described how the approximation may be post-processed in the neighborhood of a re-entrant corner singularity in order to obtain an improved and more rapidly converging representation since usually near the singularity the expansions converge very slowly.

The idea of matching eigenfunction expansions by decomposing the region has been done by different authors in various ways other than using the biorthogonality conditions. For example, using inversion or collocation techniques, Dagan, Weinbaum, and Pfeffer [2] used matching eigenfunction expansions to solve the axisymmetric Stokes flow through a pore in a wall. The flow field is partitioned into two regions: an infinite half-space outside the pore and a cylindrical volume bounded by the walls of the pore. The solution they obtained led to Fourier, Fourier-Bessel, and Dini series which were all invertible over the common matching interface. A system of linear equations was obtained which was solved by successive truncations.

All the above sources cannot treat the flow through a channel bend. This is because biorthogonality conditions for a cylindrical sector are needed at the curved region. Such conditions were developed recently by Khuri [13], which we shall utilize in our analysis. We shall also illustrate the matching method for different coordinate systems.

2. Formulation. A two-dimensional infinite channel $\Omega$ with a bend is filled with a viscous incompressible fluid (see Fig. 1). The fluid is set into motion by a fixed flow rate at infinity. In the absence of inertial terms we have Stokes flow; thus we are considering solving the biharmonic problem:

$$
\begin{gathered}
\nabla^{4} \Psi=0 \quad \text { in } \Omega, \\
\Psi=0 \quad \text { on } \Gamma_{1}, \quad \Psi=1 \quad \text { on } \Gamma_{2}, \\
\frac{\partial \Psi}{\partial n}=0 \quad \text { on } \Gamma_{1} \cup \Gamma_{2}
\end{gathered}
$$

where $\Psi$ is the stream function and $n$ is the direction normal to the boundaries. In (2.2)-(2.3) the no-slip conditions are imposed on the rigid boundaries of the region $\Omega$.

From the symmetry of the region we are only concerned here with subregions (I) and (II) shown in Fig. 1.

The derivatives of $\Psi_{\mathrm{I}}$ give the velocity components in the $X$ and $Y$ directions respectively as

$$
u=\frac{\partial \Psi_{\mathrm{I}}}{\partial Y}, \quad v=-\frac{\partial \Psi_{\mathrm{I}}}{\partial X} .
$$

The derivatives of $\Psi_{\text {II }}$ give the velocity components

$$
v_{\theta}=\frac{\partial \Psi_{\mathrm{II}}}{\partial r}, \quad v_{r}=\frac{-1}{r} \frac{\partial \Psi_{11}}{\partial \theta}
$$

where $v_{r}$ and $v_{\theta}$ are the radial and peripheral velocity components respectively.

Nondimensionalize all lengths by the minimum radius of curvature such that the constant gap width is $a-1>0$. 


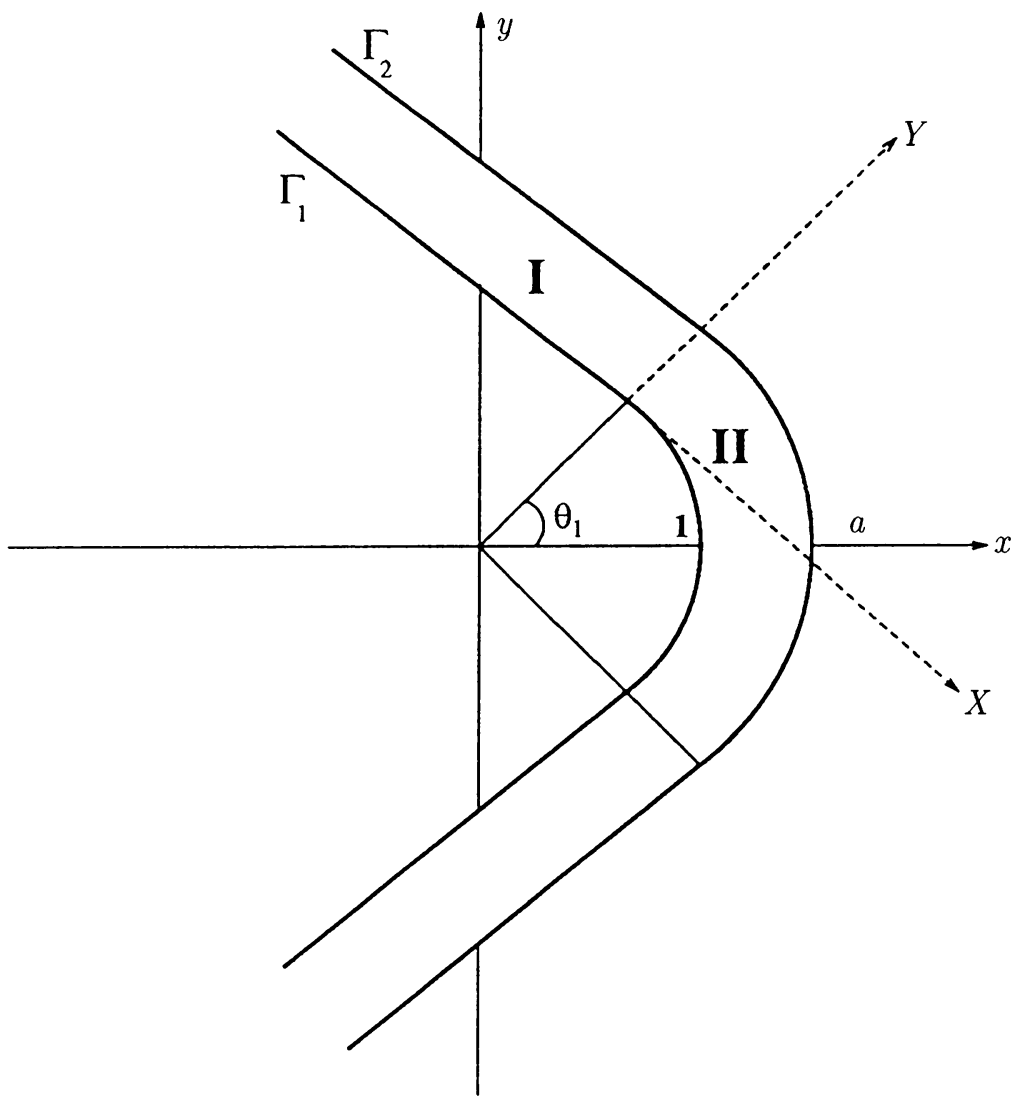

FrG. 1. Coordinate system of the channel with a bend

In particular, the solutions $\Psi_{\mathrm{I}}$ and $\Psi_{\mathrm{II}}$ of Eqs. (2.1)-(2.3) in subregions (I) and (II) respectively, satisfy the following equations:

$$
\nabla^{4} \Psi_{\mathrm{I}}(X, Y)=\left(\frac{\partial^{2}}{\partial X^{2}}+\frac{\partial^{2}}{\partial Y^{2}}\right)^{2} \Psi_{\mathrm{I}}(X, Y)=0
$$

in

$$
\mathcal{V}_{\mathrm{I}}=\{X, Y:-\infty<X \leq 0,0 \leq Y \leq a-1\}
$$

with boundary conditions

$$
\Psi_{\mathrm{I}}(X, 0)=\Psi_{\mathrm{I}}(X, a-1)-1=\frac{\partial \Psi_{\mathrm{I}}}{\partial Y}(X, 0)=\frac{\partial \Psi_{\mathrm{I}}}{\partial Y}(X, a-1)=0
$$

and

$$
\nabla^{4} \Psi_{\mathrm{II}}(r, \theta)=\left(\frac{\partial^{2}}{\partial r^{2}}+\frac{1}{r} \frac{\partial}{\partial r}+\frac{1}{r^{2}} \frac{\partial^{2}}{\partial \theta^{2}}\right)^{2} \Psi_{\mathrm{II}}(r, \theta)=0
$$

in

$$
\mathcal{V}_{\mathrm{II}}=\left\{r, \theta: 1 \leq r \leq a,-\theta_{1} \leq \theta \leq \theta_{1}\right\}
$$


with boundary conditions

$$
\Psi_{\mathrm{II}}(1, \theta)=\Psi_{\mathrm{II}}(a, \theta)-1=\frac{\partial \Psi_{\mathrm{II}}}{\partial r}(1, \theta)=\frac{\partial \Psi_{\mathrm{II}}}{\partial r}(a, \theta)=0 .
$$

The general solution for region (I) was developed by Joseph [5],

$$
\Psi_{\mathrm{I}}(X, Y)=\Psi_{0}(Y)+\sum_{-\infty}^{\infty}\left\{\frac{C_{n}}{b^{2} S_{n}^{2}} e^{b S_{n} X} \tilde{\phi}_{1}^{(n)}(Y)+\frac{D_{n}}{b^{2} P_{n}^{2}} e^{b P_{n} X} \hat{\phi}_{1}^{(n)}(Y)\right\}
$$

where (2.10) and the parabolic flow $\Psi_{0}(Y)$, given by

$$
\Psi_{0}(Y)=-\frac{2}{(a-1)^{3}} Y^{3}+\frac{3}{(a-1)^{2}} Y^{2}
$$

satisfy (2.6) and the boundary conditions (2.7). Also we have

$$
\begin{aligned}
& \tilde{\phi}_{1}^{(n)}(Y)=S_{n} \sin S_{n} \cos \left[S_{n}(b Y-1)\right]-S_{n}(b Y-1) \cos S_{n} \sin \left[S_{n}(b Y-1)\right], \\
& \hat{\phi}_{1}^{(n)}(Y)=P_{n} \cos P_{n} \sin \left[P_{n}(b Y-1)\right]-P_{n}(b Y-1) \sin P_{n} \cos \left[P_{n}(b Y-1)\right],
\end{aligned}
$$

where

$$
b=\frac{2}{a-1} .
$$

The eigenfunctions $\tilde{\phi}_{1}^{(n)}$ and $\hat{\phi}_{1}^{(n)}$ satisfy the following boundary conditions:

$$
\begin{aligned}
& \tilde{\phi}_{1}^{(n)}(0)=\tilde{\phi}_{1}^{(n)}(a-1)=\frac{d \tilde{\phi}_{1}^{(n)}}{d Y}(0)=\frac{d \tilde{\phi}_{1}^{(n)}}{d Y}(a-1)=0, \\
& \hat{\phi}_{1}^{(n)}(0)=\hat{\phi}_{1}^{(n)}(a-1)=\frac{d \hat{\phi}_{1}^{(n)}}{d Y}(0)=\frac{d \hat{\phi}_{1}^{(n)}}{d Y}(a-1)=0 .
\end{aligned}
$$

The eigenvalues $S_{n}, n=1,2, \ldots$ satisfy

$$
\sin 2 S+2 S=0
$$

and the eigenvalues $P_{n}, n=1,2, \ldots$ satisfy

$$
\sin 2 P-2 P=0 \text {. }
$$

These eigenvalues are symmetrically located in the complex $S$ and $P$ planes respectively; we choose $S_{n}, P_{n}, n>0$, to be the first quadrant roots, which are arranged in a sequence corresponding to increasing size of their real part, and define

$$
\begin{array}{ll}
S_{-n}=\bar{S}_{n} & (n=1,2,3, \ldots), \\
P_{-n}=\bar{P}_{n} \quad(n=1,2,3, \ldots),
\end{array}
$$

where the overbar designates complex conjugate. Then

$$
\begin{aligned}
& \tilde{\phi}_{1}^{(-n)}(Y)=\overline{\tilde{\phi}_{1}^{(n)}(Y)} \quad(n=1,2,3, \ldots), \\
& \hat{\phi}_{1}^{(-n)}(Y)=\overline{\hat{\phi}_{1}^{(n)}(Y)} \quad(n=1,2,3, \ldots)
\end{aligned}
$$

are the eigenfunctions corresponding to the eigenvalues $\lambda_{-n}$. Since the boundary data given in (2.7) and (2.9) are real, then

$$
C_{-n}=\bar{C}_{n} ; \quad D_{-n}=\bar{D}_{n} .
$$


The following property of biorthogonality, see Joseph [5], is satisfied by the eigenfunctions and adjoint eigenfunctions

$$
\int_{0}^{a-1}\left[\tilde{\psi}_{1}^{(m)}(Y), \tilde{\psi}_{2}^{(m)}(Y)\right]\left(\begin{array}{cc}
0 & -1 \\
1 & 2
\end{array}\right)\left[\begin{array}{c}
\tilde{\phi}_{1}^{(n)}(Y) \\
\tilde{\phi}_{2}^{(n)}(Y)
\end{array}\right] d Y=k_{n} \delta_{m n}
$$

and

$$
\int_{0}^{a-1}\left[\hat{\psi}_{1}^{(m)}(Y), \hat{\psi}_{2}^{(m)}(Y)\right]\left(\begin{array}{cc}
0 & -1 \\
1 & 2
\end{array}\right)\left[\begin{array}{c}
\hat{\phi}_{1}^{(n)}(Y) \\
\hat{\phi}_{2}^{(n)}(Y)
\end{array}\right] d Y=\hat{k}_{n} \delta_{m n}
$$

The functions $\tilde{\psi}_{1}^{(n)}$ and $\tilde{\psi}_{2}^{(n)}$ are related by

$$
\frac{d^{2} \tilde{\psi}_{1}^{(n)}}{d Y^{2}}=-b^{2} S_{n}^{2} \tilde{\psi}_{2}^{(n)}
$$

and the functions $\hat{\psi}_{1}^{(n)}$ and $\hat{\psi}_{2}^{(n)}$ are related by

$$
\frac{d^{2} \hat{\psi}_{1}^{(n)}}{d Y^{2}}=-b^{2} P_{n}^{2} \hat{\psi}_{2}^{(n)}
$$

Also

$$
\begin{aligned}
\frac{d^{2} \tilde{\phi}_{1}^{(n)}}{d Y^{2}} & =b^{2} S_{n}^{2} \tilde{\phi}_{2}^{(n)} \\
\frac{d^{2} \hat{\phi}_{1}^{(n)}}{d Y^{2}} & =b^{2} P_{n}^{2} \hat{\phi}_{2}^{(n)}
\end{aligned}
$$

The adjoint eigenfunctions are given by

$$
\begin{gathered}
\tilde{\psi}_{1}^{(m)}(Y)=\tilde{\phi}_{1}^{(m)}(Y)-2 \cos S_{m} \cos \left[S_{m}(b Y-1)\right] \\
\tilde{\psi}_{2}^{(m)}(Y)=\tilde{\phi}_{1}^{(m)}(Y) \\
\hat{\psi}_{2}^{(m)}(Y)=\hat{\phi}_{1}^{(m)}(Y)
\end{gathered}
$$

and

$$
\hat{\psi}_{1}^{(m)}(Y)=\hat{\phi}_{1}^{(m)}(Y)+2 \sin P_{m} \sin \left[P_{m}(b Y-1)\right] .
$$

The constants $k_{n}$ and $\hat{k}_{n}$ in the biorthogonality conditions (2.17)-(2.18) are given by

$$
\begin{aligned}
& k_{n}=-2(a-1) \cos ^{4} S_{n}, \\
& \hat{k}_{n}=-2(a-1) \sin ^{4} P_{n} .
\end{aligned}
$$

As for the sector region (II), the general solution satisfying (2.8) and (2.9) is (see [13] for details)

$$
\Psi_{\mathrm{II}}(r, \theta)=\widehat{\Psi}_{0}(r)+\sum_{-\infty}^{\infty} F_{n} \cos \left(\lambda_{n} \theta\right) \phi_{1}^{(n)}(r) .
$$

We note that the complete solution $\Psi_{\mathrm{II}}(r, \theta)$ given in (2.25) contains $\sin \left(\lambda_{n} \theta\right)$ terms, but due to the symmetry with respect to $\theta$ in subregion (II) (see Fig. 1), only the cosine term is retained. 
In $(2.25) \widehat{\Psi}_{0}(r)$ is a concentric flow satisfying (2.8) and the boundary conditions given in $(2.9)$ :

$$
\begin{array}{r}
\widehat{\Psi}_{0}(r)=\frac{1}{\left[\left(a^{2}-1\right)^{2}-4 a^{2} \log ^{2} a\right]}\left\{\left(2 a^{2} \log a+a^{2}-1\right)\left(r^{2}-1\right)\right. \\
\left.-4 a^{2} \log a \log r-2\left(a^{2}-1\right)\left(r^{2} \log r\right)\right\} .
\end{array}
$$

Also

$$
\phi_{1}^{(n)}(r)=a_{n} r^{\lambda_{n}}+b_{n} r^{-\lambda_{n}}+c_{n} r^{2-\lambda_{n}}+d_{n} r^{2+\lambda_{n}} .
$$

The coefficients $a_{n}, b_{n}, c_{n}, d_{n}$ and the corresponding eigenvalues $\lambda_{n}$ are also given in [13]. In particular, the eigenvalues $\lambda_{n}$ satisfy

$$
\begin{gathered}
\sin \hat{\lambda}_{n}= \pm \beta \hat{\lambda}_{n}, \\
\hat{\lambda}_{n}=(i \log a) \lambda_{n}, \quad \beta=\frac{1}{2 \log a}\left(a-\frac{1}{a}\right) .
\end{gathered}
$$

These eigenvalues are symmetrically located in the complex $\lambda$ plane; we choose $\lambda_{n}$, $n>0$, to be the first quadrant roots, which are arranged in a sequence corresponding to increasing size of their real part, and define

$$
\lambda_{-n}=\bar{\lambda}_{n} \quad(n=1,2,3, \ldots),
$$

where the overbar designates complex conjugate. Then

$$
\phi_{1}^{(-n)}(r)=\bar{\phi}_{1}^{(n)}(r) \quad(n=1,2,3, \ldots)
$$

is the eigenfunction corresponding to the eigenvalue $\lambda_{-n}$. Since the edge data given in (2.7) and (2.9) are real, then

$$
F_{-n}=\bar{F}_{n}
$$

3. Matching eigenfunction expansions. We must now match the two solutions given in (2.10) and (2.25). We shall require that the stream function and its first three partial derivatives with respect to $X$ are continuous across the interface (i.e., when $\theta=$ $\theta_{1}$ and $X=0$ ) between region (I) and region (II). We impose $C^{3}$ continuity of the stream function across subregion interfaces in order to determine the coefficients in the eigenfunction expansions. We require

$$
\begin{aligned}
\Psi_{\mathrm{I}}(0, Y) & =\Psi_{\mathrm{II}}\left(r, \theta_{1}\right), \\
\frac{\partial \Psi_{\mathrm{I}}}{\partial X}(0, Y) & =\frac{\partial \Psi_{\mathrm{II}}}{\partial X}\left(r, \theta_{1}\right), \\
\frac{\partial^{2} \Psi_{\mathrm{I}}}{\partial X^{2}}(0, Y) & =\frac{\partial^{2} \Psi_{\mathrm{II}}}{\partial X^{2}}\left(r, \theta_{1}\right), \\
\frac{\partial^{3} \Psi_{\mathrm{I}}}{\partial X^{3}}(0, Y) & =\frac{\partial^{3} \Psi_{\mathrm{II}}}{\partial X^{3}}\left(r, \theta_{1}\right),
\end{aligned}
$$

where

$$
0 \leq Y \leq a-1 \quad \text { and } \quad 1 \leq r \leq a .
$$


In Eqs. (3.28)-(3.31), across the interface, we have

$$
r=Y+1
$$

To prepare for the computation of the equations given in (3.28)-(3.31) we require first some partial derivatives across the interface. From Fig. 1 we have

$$
\begin{aligned}
& X=x^{\prime} \cos \left(\frac{\pi}{2}-\theta_{1}\right)-y^{\prime} \sin \left(\frac{\pi}{2}-\theta_{1}\right) \\
& Y=x^{\prime} \sin \left(\frac{\pi}{2}-\theta_{1}\right)+y^{\prime} \cos \left(\frac{\pi}{2}-\theta_{1}\right)
\end{aligned}
$$

where

$$
\begin{aligned}
& x=x^{\prime}+\cos \theta_{1}, \\
& y=y^{\prime}+\sin \theta_{1} .
\end{aligned}
$$

Substituting (3.34) into Eq. (3.33) and making use of the following trigonometric identity,

$$
\cos (A+B)=\cos A \cos B-\sin A \sin B,
$$

we get

$$
X=x \cos \left(\frac{\pi}{2}-\theta_{1}\right)-y \sin \left(\frac{\pi}{2}-\theta_{1}\right)
$$

Similarly, now using the trigonometric identity

$$
\sin (A+B)=\sin A \cos B+\sin B \cos A,
$$

we get

$$
Y=x \sin \left(\frac{\pi}{2}-\theta_{1}\right)+y \cos \left(\frac{\pi}{2}-\theta_{1}\right)-1 .
$$

We have thus the following:

$$
\begin{aligned}
& X=r \cos \theta \cos \left(\frac{\pi}{2}-\theta_{1}\right)-r \sin \theta \sin \left(\frac{\pi}{2}-\theta_{1}\right) \\
& Y=r \cos \theta \sin \left(\frac{\pi}{2}-\theta_{1}\right)+r \sin \theta \cos \left(\frac{\pi}{2}-\theta_{1}\right)-1
\end{aligned}
$$

where we replaced $x$ and $y$ by

$$
\begin{aligned}
& x=r \cos \theta, \\
& y=r \sin \theta .
\end{aligned}
$$

Using the identities (3.35)-(3.36), Eq. (3.37) can be simplified to

$$
\begin{aligned}
& X=r \cos \left(\theta-\theta_{1}+\frac{\pi}{2}\right) \\
& Y=r \sin \left(\theta-\theta_{1}+\frac{\pi}{2}\right)-1 .
\end{aligned}
$$

Differentiating both equations in (3.38) with respect to $X$ we get

$$
\begin{aligned}
& 1=\frac{\partial r}{\partial X} \cos \left(\theta-\theta_{1}+\frac{\pi}{2}\right)-r \frac{\partial \theta}{\partial X} \sin \left(\theta-\theta_{1}+\frac{\pi}{2}\right) \\
& 0=\frac{\partial r}{\partial X} \sin \left(\theta-\theta_{1}+\frac{\pi}{2}\right)+r \frac{\partial \theta}{\partial X} \cos \left(\theta-\theta_{1}+\frac{\pi}{2}\right) .
\end{aligned}
$$


Across the interface of regions (I) and (II), (i.e., when $\left.\theta=\theta_{1}, X=0\right)$, Eqs. (3.39) reduce to

$$
\begin{aligned}
& \frac{\partial \theta}{\partial X}=-\frac{1}{r} \\
& \frac{\partial r}{\partial X}=0 .
\end{aligned}
$$

Differentiating the second equation in (3.38) twice with respect to $X$, then using the chain rule, we have

$$
0=\left[\frac{\partial^{2} r}{\partial X^{2}}-r\left(\frac{\partial \theta}{\partial X}\right)^{2}\right] \sin \left(\theta-\theta_{1}+\frac{\pi}{2}\right)+\left[r \frac{\partial^{2} \theta}{\partial X^{2}}+2 \frac{\partial r}{\partial X} \frac{\partial \theta}{\partial X}\right] \cos \left(\theta-\theta_{1}+\frac{\pi}{2}\right)
$$

Similarly, differentiating the first equation in (3.38) twice with respect to $X$ and then using the chain rule, we get

$$
0=\left[\frac{\partial^{2} r}{\partial X^{2}}-r\left(\frac{\partial \theta}{\partial X}\right)^{2}\right] \cos \left(\theta-\theta_{1}+\frac{\pi}{2}\right)-\left[r \frac{\partial^{2} \theta}{\partial X^{2}}+2 \frac{\partial r}{\partial X} \frac{\partial \theta}{\partial X}\right] \sin \left(\theta-\theta_{1}+\frac{\pi}{2}\right)
$$

Making use of (3.40), Eqs. (3.41)-(3.42) across the interface of regions (I) and (II) reduce to

$$
\begin{aligned}
& \frac{\partial^{2} r}{\partial X^{2}}=\frac{1}{r}, \\
& \frac{\partial^{2} \theta}{\partial X^{2}}=0 .
\end{aligned}
$$

In the same way, if we differentiate both sides of Eqs. (3.38) three times with respect to $X$, taking into account Eqs. (3.40) and (3.43), then the equations across the interface of regions (I) and (II) reduce to

$$
\begin{aligned}
& \frac{\partial^{3} r}{\partial X^{3}}=0 \\
& \frac{\partial^{3} \theta}{\partial X^{3}}=\frac{2}{r^{3}}
\end{aligned}
$$

From Eqs. (3.40), (3.43), and (3.44), we have the following values of partial derivatives across the interface (where $\theta=\theta_{1}$ and $X=0$ ):

$$
\left\{\begin{aligned}
\frac{\partial r}{\partial X} & =\frac{\partial^{2} \theta}{\partial X^{2}}=\frac{\partial^{3} r}{\partial X^{3}}=0 \\
\frac{\partial \theta}{\partial X} & =-\frac{1}{r} \\
\frac{\partial^{2} r}{\partial X^{2}} & =\frac{1}{r} \\
\frac{\partial^{3} \theta}{\partial X^{3}} & =\frac{2}{r^{3}}
\end{aligned}\right.
$$


Using (3.45) we can now compute the partial derivatives of $\Psi$ with respect to $X$; we have

$$
\frac{\partial \Psi}{\partial X}=\frac{\partial \Psi}{\partial r} \frac{\partial r}{\partial X}+\frac{\partial \Psi}{\partial \theta} \frac{\partial \theta}{\partial X}
$$

Across the interface this reduces to

$$
\frac{\partial \Psi}{\partial X}=-\frac{1}{r} \frac{\partial \Psi}{\partial \theta}
$$

For the second partial derivative,

$$
\frac{\partial^{2} \Psi}{\partial X^{2}}=\frac{\partial^{2} \Psi}{\partial X \partial r} \frac{\partial r}{\partial X}+\frac{\partial \Psi}{\partial r} \frac{\partial^{2} r}{\partial X^{2}}+\frac{\partial^{2} \Psi}{\partial X \partial \theta} \frac{\partial \theta}{\partial X}+\frac{\partial \Psi}{\partial \theta} \frac{\partial^{2} \theta}{\partial X^{2}}
$$

Using (3.45) and (3.47), across the interface, the last equation reduces to

$$
\frac{\partial^{2} \Psi}{\partial X^{2}}=\frac{\partial \Psi}{\partial r} \frac{\partial^{2} r}{\partial X^{2}}+\frac{\partial^{2} \Psi}{\partial \theta^{2}}\left(\frac{\partial \theta}{\partial X}\right)^{2}=\frac{1}{r} \frac{\partial \Psi}{\partial r}+\frac{1}{r^{2}} \frac{\partial^{2} \Psi}{\partial \theta^{2}}
$$

For the third partial derivative,

$$
\begin{aligned}
\frac{\partial^{3} \Psi}{\partial X^{3}}=\frac{\partial^{2} \Psi}{\partial X \partial r} \frac{\partial^{2} r}{\partial X^{2}} & +\frac{\partial^{3} \Psi}{\partial X^{2} \partial r} \frac{\partial r}{\partial X}+\frac{\partial \Psi}{\partial r} \frac{\partial^{3} r}{\partial X^{3}}+\frac{\partial^{2} \Psi}{\partial X \partial r} \frac{\partial^{2} r}{\partial X^{2}}+\frac{\partial^{2} \Psi}{\partial X \partial \theta} \frac{\partial^{2} \theta}{\partial X^{2}} \\
& +\frac{\partial^{3} \Psi}{\partial X^{2} \partial \theta} \frac{\partial \theta}{\partial X}+\frac{\partial \Psi}{\partial \theta} \frac{\partial^{3} \theta}{\partial X^{3}}+\frac{\partial^{2} \Psi}{\partial X \partial \theta} \frac{\partial^{2} \theta}{\partial X^{2}}
\end{aligned}
$$

but we have the following partial derivatives:

$$
\begin{aligned}
\frac{\partial^{2} \Psi}{\partial X \partial r}= & \frac{\partial^{2} \Psi}{\partial r^{2}} \frac{\partial r}{\partial X}+\frac{\partial^{2} \Psi}{\partial r \partial \theta} \frac{\partial \theta}{\partial X} \\
\frac{\partial^{2} \Psi}{\partial X \partial \theta}= & \frac{\partial^{2} \Psi}{\partial \theta^{2}} \frac{\partial \theta}{\partial X}+\frac{\partial^{2} \Psi}{\partial \theta \partial r} \frac{\partial r}{\partial X} \\
\frac{\partial^{3} \Psi}{\partial X^{2} \partial \theta}= & \frac{\partial^{2} \Psi}{\partial \theta^{2}} \frac{\partial^{2} \theta}{\partial X^{2}}+\left(\frac{\partial^{3} \Psi}{\partial \theta^{3}} \frac{\partial \theta}{\partial X}+\frac{\partial^{3} \Psi}{\partial r \partial \theta^{2}} \frac{\partial r}{\partial X}\right) \frac{\partial \theta}{\partial X}+\frac{\partial^{2} \Psi}{\partial \theta \partial r} \frac{\partial^{2} r}{\partial X^{2}} \\
& +\left(\frac{\partial^{3} \Psi}{\partial \theta \partial r^{2}} \frac{\partial r}{\partial X}+\frac{\partial^{3} \Psi}{\partial r \partial \theta^{2}} \frac{\partial \theta}{\partial X}\right) \frac{\partial r}{\partial X}
\end{aligned}
$$

Across the interface they reduce to

$$
\begin{aligned}
\frac{\partial^{2} \Psi}{\partial X \partial r} & =-\frac{1}{r} \frac{\partial^{2} \Psi}{\partial \theta \partial r} \\
\frac{\partial^{2} \Psi}{\partial X \partial \theta} & =-\frac{1}{r} \frac{\partial^{2} \Psi}{\partial \theta^{2}}+\frac{\partial^{2} \Psi}{\partial \theta \partial r} \frac{\partial r}{\partial X} \\
\frac{\partial^{3} \Psi}{\partial X^{2} \partial \theta} & =\frac{1}{r^{2}} \frac{\partial^{3} \Psi}{\partial \theta^{3}}+\frac{1}{r} \frac{\partial^{2} \Psi}{\partial r \partial \theta}
\end{aligned}
$$

So across the interface, the third partial derivative in (3.50) reduces to

$$
\begin{aligned}
\frac{\partial^{3} \Psi}{\partial X^{3}} & =2 \frac{\partial^{2} \Psi}{\partial X \partial r} \frac{\partial^{2} r}{\partial X^{2}}+\frac{\partial^{3} \Psi}{\partial X^{2} \partial \theta} \frac{\partial \theta}{\partial X}+\frac{\partial \Psi}{\partial \theta} \frac{\partial^{3} \theta}{\partial X^{3}} \\
& =-\frac{2}{r^{2}} \frac{\partial^{2} \Psi}{\partial r \partial \theta}-\frac{1}{r^{3}} \frac{\partial^{3} \Psi}{\partial \theta^{3}}-\frac{1}{r^{2}} \frac{\partial^{2} \Psi}{\partial r \partial \theta}+\frac{2}{r^{3}} \frac{\partial \Psi}{\partial \theta}
\end{aligned}
$$


Across the interface we have thus the following partial derivatives:

$$
\begin{aligned}
\frac{\partial \Psi}{\partial X} & =-\frac{1}{r} \frac{\partial \Psi}{\partial \theta}, \\
\frac{\partial^{2} \Psi}{\partial X^{2}} & =\frac{1}{r} \frac{\partial \Psi}{\partial r}+\frac{1}{r^{2}} \frac{\partial^{2} \Psi}{\partial \theta^{2}}, \\
\frac{\partial^{3} \Psi}{\partial X^{3}} & =-\frac{1}{r^{3}} \frac{\partial^{3} \Psi}{\partial \theta^{3}}-\frac{3}{r^{2}} \frac{\partial^{2} \Psi}{\partial r \partial \theta}+\frac{2}{r^{3}} \frac{\partial \Psi}{\partial \theta} .
\end{aligned}
$$

In particular, across the interface, the partial derivatives of $\widehat{\Psi}_{0}$ upon using (3.54) are given by

$$
\begin{aligned}
\frac{\partial \widehat{\Psi}_{0}}{\partial X} & =\frac{\partial^{3} \widehat{\Psi}_{0}}{\partial X^{3}}=0 \\
\frac{\partial \widehat{\Psi}_{0}}{\partial X} & =\frac{\partial^{2} \widehat{\Psi}_{0}}{\partial X^{2}}=\frac{\partial^{3} \widehat{\Psi}_{0}}{\partial X^{3}}=0 \\
\frac{\partial^{2} \widehat{\Psi}_{0}}{\partial X^{2}} & =\frac{1}{r} \frac{\partial \widehat{\Psi}_{0}}{\partial r}
\end{aligned}
$$

Substituting the eigenfunction expansions given in $(2.10),(2.25)$ into the matching conditions (3.28)-(3.31) and making use of Eqs. (3.54) and (3.55) we get the following matching equations:

$$
\begin{gathered}
0=\widehat{\Psi}_{0}(r)-\Psi_{0}(Y)+\sum_{-\infty}^{\infty} F_{n} \cos \left(\lambda_{n} \theta_{1}\right) \phi_{1}^{(n)}(r)-\sum_{-\infty}^{\infty}\left\{\frac{C_{n}}{b^{2} S_{n}^{2}} \tilde{\phi}_{1}^{(n)}(Y)+\frac{D_{n}}{b^{2} P_{n}^{2}} \hat{\phi}_{1}^{(n)}(Y)\right\} \\
\begin{array}{c}
0 \sum_{-\infty}^{\infty} F_{n} \lambda_{n} \sin \left(\lambda_{n} \theta_{1}\right)\left(\frac{1}{r} \phi_{1}^{(n)}(r)\right)-\sum_{-\infty}^{\infty}\left\{\frac{C_{n}}{b S_{n}} \tilde{\phi}_{1}^{(n)}(Y)+\frac{D_{n}}{b P_{n}} \hat{\phi}_{1}^{(n)}(Y)\right\} \\
0=\frac{1}{r} \frac{d \widehat{\Psi}_{0}(r)}{d r}+\sum_{-\infty}^{\infty} F_{n} \cos \left(\lambda_{n} \theta_{1}\right)\left(\frac{1}{r} \frac{d \phi_{1}^{(n)}(r)}{d r}-\frac{1}{r^{2}} \lambda_{n}^{2} \phi_{1}^{(n)}(r)\right) \\
0=\sum_{-\infty}^{\infty} F_{n} \sin \left(\lambda_{n} \theta_{1}\right)\left(\frac{3}{r^{2}} \lambda_{n} \frac{d \phi_{1}^{(n)}(r)}{d r}-\frac{1}{r^{3}} \lambda_{n}^{3} \phi_{1}^{(n)}(r)-\frac{2}{r^{3}} \lambda_{n} \phi_{1}^{(n)}(r)\right) \\
-\sum_{-\infty}^{\infty}\left[C_{n} b \tilde{\phi}_{n}^{(n)}(Y)+\tilde{\phi}_{1}^{(n)}(Y)+\hat{\phi}_{1}^{(n)}(Y)\right]
\end{array}
\end{gathered}
$$

where $r$ and $Y$ in Eqs. (3.56)-(3.59) are on the interface related by

$$
r=Y+1 \text {. }
$$

In the next section we describe how the coefficients $C_{n}, D_{n}$, and $F_{n}$ are determined using biorthogonality conditions satisfied by the Papkovich-Fadle eigenfunctions. 
4. Biorthogonality series solution. We can find the coefficients $C_{n}, D_{n}$, and $F_{n}$ by application of the biorthogonality conditions to the matching conditions given in Eqs. (3.56)-(3.59). The biorthogonality conditions for general fourth-order differential equations were derived by Khuri (1994) [13]; these conditions for the sectorial geometry are briefly described as follows.

To prepare for the application of the biorthogonality condition we need first to express the matching conditions given in (3.56)-(3.59) in vector form. Differentiating both sides of Eq. (3.56) twice and using that

$$
\begin{aligned}
\frac{d^{2} \tilde{\phi}_{1}^{(n)}}{d Y^{2}} & =b^{2} S_{n}^{2} \tilde{\phi}_{2}^{(n)}, \\
\frac{d^{2} \hat{\phi}_{1}^{(n)}}{d Y^{2}} & =b^{2} P_{n}^{2} \hat{\phi}_{2}^{(n)},
\end{aligned}
$$

then we can couple Eqs. (3.56) and (3.58) as follows:

$$
\begin{gathered}
\left(\begin{array}{l}
0 \\
0
\end{array}\right)=\left(\left[\begin{array}{c}
\frac{1}{r} \frac{d \hat{\Psi}_{0}}{d r} \\
\left.\frac{d^{2} \widehat{\Psi}_{0}}{d r^{2}}-\frac{d^{2} \Psi_{0}}{d Y^{2}}\right]_{X=0}
\end{array}\right)+\sum_{-\infty}^{\infty} F_{n} \cos \left(\lambda_{n} \theta_{1}\right)\left(\begin{array}{c}
\frac{1}{r} \frac{d \phi_{1}^{(n)}(r)}{d r}-\frac{1}{r^{2}} \lambda_{n}^{2} \phi_{1}^{(n)}(r) \\
\frac{d^{2} \phi_{1}^{(n)}(r)}{d r^{2}}
\end{array}\right)\right. \\
-\sum_{-\infty}^{\infty}\left\{C_{n}\left(\begin{array}{c}
\tilde{\phi}_{1}^{(n)}(Y) \\
\tilde{\phi}_{2}^{(n)}(Y)
\end{array}\right)+D_{n}\left(\begin{array}{c}
\hat{\phi}_{1}^{(n)}(Y) \\
\hat{\phi}_{2}^{(n)}(Y)
\end{array}\right)\right\} .
\end{gathered}
$$

Now apply the operator

$$
\int_{0}^{a-1}\left[\tilde{\psi}_{1}^{(m)}(Y), \tilde{\psi}_{2}^{(m)}(Y)\right]\left(\begin{array}{cc}
0 & -1 \\
1 & 2
\end{array}\right)[\cdot] d Y
$$

to both sides of Eq. (4.62) and let

$$
\begin{array}{r}
U_{m n}=\int_{0}^{a-1}\left[\tilde{\psi}_{1}^{(m)}(Y), \tilde{\psi}_{2}^{(m)}(Y)\right]\left(\begin{array}{cc}
0 & -1 \\
1 & 2
\end{array}\right)\left\{\left(\begin{array}{c}
\frac{1}{Y+1} \frac{d \phi_{1}^{(n)}(Y+1)}{d r} \\
\frac{d^{2} \phi_{1}^{(n)}(Y+1)}{d r^{2}}
\end{array}\right)\right. \\
\left.-\left(\begin{array}{c}
\left.\left.\frac{1}{(Y+1)^{2}} \lambda_{n}^{2} \phi_{1}^{(n)}(Y+1)\right)\right\} \\
0
\end{array}\right)\right\} Y, \\
Q_{m}=\int_{0}^{a-1}\left[\tilde{\psi}_{1}^{(m)}(Y), \tilde{\psi}_{2}^{(m)}(Y)\right]\left(\begin{array}{cc}
0 & -1 \\
1 & 2
\end{array}\right)\left[\begin{array}{c}
\frac{1}{r} \frac{d \widehat{\Psi}_{0}(r)}{d r} \\
\left(\frac{d^{2} \widehat{\Psi}_{0}}{d r^{2}}-\frac{d^{2} \Psi_{0}}{d Y^{2}}\right)_{X=0}
\end{array}\right] d Y .
\end{array}
$$

Also, the following integral vanishes:

$$
\int_{0}^{a-1}\left[\tilde{\psi}_{1}^{(m)}(Y), \tilde{\psi}_{2}^{(m)}(Y)\right]\left(\begin{array}{cc}
0 & -1 \\
1 & 2
\end{array}\right)\left[\begin{array}{l}
\hat{\phi}_{1}^{(n)}(Y) \\
\hat{\phi}_{2}^{(n)}(Y)
\end{array}\right] d Y=0
$$

Expanding the integrand in (4.65) gives

$$
-\hat{\phi}_{2}^{(n)}(Y) \tilde{\psi}_{1}^{(m)}(Y)+\left(\hat{\phi}_{1}^{(n)}(Y)+2 \hat{\phi}_{2}^{(n)}(Y)\right) \tilde{\psi}_{2}^{(m)}(Y) .
$$

Making the change of variables $Z=b Y-1$, this integrand becomes an odd function of $Z$ integrated on $[-1,1]$. This can easily be seen by substituting the definitions of 
the eigenfunctions and adjoint eigenfunctions (2.12), (2.21), and (2.22) into this integrand, simplifying it through multiplication. Hence by making use of the biorthogonality condition we get

$$
0=Q_{m}-k_{m} C_{m}+\sum_{-\infty}^{\infty} F_{n} U_{m n} \cos \left(\lambda_{n} \theta_{1}\right) .
$$

Now apply the operator

$$
\int_{0}^{a-1}\left[\hat{\psi}_{1}^{(m)}(Y), \hat{\psi}_{2}^{(m)}(Y)\right]\left(\begin{array}{cc}
0 & -1 \\
1 & 2
\end{array}\right)[\cdot] d Y
$$

to both sides of Eq. (4.62) and let

$$
\begin{array}{r}
\widehat{U}_{m n}=\int_{0}^{a-1}\left[\tilde{\psi}_{1}^{(m)}(Y), \hat{\psi}_{2}^{(m)}(Y)\right]\left(\begin{array}{cc}
0 & -1 \\
1 & 2
\end{array}\right)\left\{\left(\begin{array}{c}
\frac{1}{Y+1} \frac{d \phi_{1}^{(n)}(Y+1)}{d r} \\
\left.\frac{d^{2} \phi_{1}^{(n)}(Y+1)}{d r^{2}}\right)
\end{array}\right)\right. \\
\left.-\left(\begin{array}{c}
\left.\left.\frac{1}{(Y+1)^{2}} \lambda_{n}^{2} \phi_{1}^{(n)}(Y+1)\right)\right\} \\
0
\end{array}\right)\right\} d Y, \\
\widehat{Q}_{m}=\int_{0}^{a-1}\left[\hat{\psi}_{1}^{(m)}(Y), \hat{\psi}_{2}^{(m)}(Y)\right]\left(\begin{array}{cc}
0 & -1 \\
1 & 2
\end{array}\right)\left[\begin{array}{c}
\frac{1}{r} \frac{d \widehat{\Psi}_{0}(r)}{d r} \\
\left(\frac{d^{2} \widehat{\Psi}_{0}}{d r^{2}}-\frac{d^{2} \Psi_{0}}{d Y^{2}}\right)_{X=0}
\end{array}\right] d Y .
\end{array}
$$

Also, the following integral vanishes since

$$
\int_{0}^{a-1}\left[\hat{\psi}_{1}^{(m)}(Y), \hat{\psi}_{2}^{(m)}(Y)\right]\left(\begin{array}{cc}
0 & -1 \\
1 & 2
\end{array}\right)\left[\begin{array}{c}
\tilde{\phi}_{1}^{(n)}(Y) \\
\tilde{\phi}_{2}^{(n)}(Y)
\end{array}\right] d Y=0
$$

Expanding the integrand in (4.69) gives

$$
-\tilde{\phi}_{2}^{(n)}(Y) \hat{\psi}_{1}^{(m)}(Y)+\left(\tilde{\phi}_{1}^{(n)}(Y)+2 \tilde{\phi}_{2}^{(n)}(Y)\right) \hat{\psi}_{2}^{(m)}(Y) .
$$

Making the change of variables $Z=b Y-1$, this integrand becomes an odd function of $Z$ integrated on $[-1,1]$. This can easily be seen by substituting the definitions of the eigenfunctions and adjoint eigenfunctions given in Eqs. (2.12), (2.21), and (2.23) into this integrand and then simplifying it through multiplication. Hence, upon using the biorthogonality condition, we get

$$
0=\widehat{Q}_{m}-\hat{k}_{m} D_{m}+\sum_{-\infty}^{\infty} F_{n} \widehat{U}_{m n} \cos \left(\lambda_{n} \theta_{1}\right) .
$$

Next, we need to couple Eqs. (3.57) and (3.59) and apply the biorthogonality condition for a sectorial cavity (see [13] for the derivation of this condition). The biorthogonality condition is given by [13]:

$$
\int_{1}^{a}\left[\psi_{1}^{(m)}(r), \psi_{2}^{(m)}(r)\right] B(r)\left[\begin{array}{l}
\phi_{1}^{(n)}(r) \\
\phi_{2}^{(n)}(r)
\end{array}\right] d r=P_{n}^{*} \delta_{m n}
$$

where

$$
\begin{array}{r}
\phi_{2}^{(n)}(r)=r \phi_{1}^{\prime \prime(n)}(r)-\frac{\mu_{n}}{2 r} \phi_{1}^{(n)}(r), \\
\psi_{1}^{(m)}(r)=\phi_{2}^{(m)}(r), \quad \text { and } \quad \psi_{2}^{(m)}(r)=\phi_{1}^{(m)}(r) .
\end{array}
$$


Here

$$
\mu_{n}=1+2 \lambda_{n}^{2}
$$

and

$$
B(r)=\left(\begin{array}{cc}
\frac{1}{2 r^{2}} & 0 \\
\frac{3}{2 r^{3}} & \frac{1}{2 r^{2}}
\end{array}\right)
$$

By (4.72) and (4.73) we have

$$
r \frac{d^{2} \phi_{1}^{(n)}(r)}{d r^{2}}=\phi_{2}^{(n)}(r)+\frac{\mu_{n}}{2 r} \phi_{1}^{(n)}(r)
$$

Then

$$
\frac{1}{r} \phi_{1}^{(n)}(r)=2 r \frac{d^{2} \phi_{1}^{(n)}(r)}{d r^{2}}-2 \phi_{2}^{(n)}(r)-\frac{2}{r} \lambda_{n}^{2} \phi_{1}^{(n)}(r)
$$

Multiplying both sides of Eq. (3.59) by $r^{3}$ and using (4.74), then Eqs. (3.57) and (3.59) can be coupled as

$$
\begin{aligned}
\left(\begin{array}{l}
0 \\
0
\end{array}\right)= & \sum_{-\infty}^{\infty} F_{n} \lambda_{n} \sin \left(\lambda_{n} \theta_{1}\right)\left\{\left(\begin{array}{c}
3 r \frac{d \phi_{1}^{(n)}}{d r}(r)-\lambda_{n}^{2} \phi_{1}^{(n)}(r) \\
2 r \frac{d^{2} \phi_{1}^{(n)}(r)}{d r^{2}}-\frac{2}{r} \lambda_{n}^{2} \phi_{1}^{(n)}(r)
\end{array}\right)-2\left(\begin{array}{c}
\phi_{1}^{(n)}(r) \\
\phi_{2}^{(n)}(r)
\end{array}\right)\right\} \\
& -\sum_{-\infty}^{\infty}\left\{C_{n}\left(\begin{array}{c}
r^{3} b S_{n} \tilde{\phi}_{1}^{(n)}(Y) \\
\frac{1}{b S_{n}} \tilde{\phi}_{1}^{(n)}(Y)
\end{array}\right)+D_{n}\left(\begin{array}{c}
r^{3} b P_{n} \hat{\phi}_{1}^{(n)}(Y) \\
\frac{1}{b P_{n}} \hat{\phi}_{1}^{(n)}(Y)
\end{array}\right)\right\}
\end{aligned}
$$

Now applying the operator

$$
\int_{1}^{a}\left[\psi_{1}^{(m)}(r), \psi_{2}^{(m)}(r)\right] B(r)[\cdot] d r
$$

to both sides of Eq. (4.75) and letting

$$
\begin{aligned}
R_{m n} & =\int_{1}^{a}\left[\psi_{1}^{(m)}(r), \psi_{2}^{(m)}(r)\right] B(r)\left[\begin{array}{c}
3 r \frac{d \phi_{1}^{(n)}}{d r}(r)-\lambda_{n}^{2} \phi_{1}^{(n)}(r) \\
2 r \frac{d^{2} \phi_{1}^{(n)}}{d r^{2}}(r)-\frac{2}{r} \lambda_{n}^{2} \phi_{1}^{(n)}(r)
\end{array}\right] d r \\
V_{m n} & =\int_{1}^{a}\left[\psi_{1}^{(m)}(r), \psi_{2}^{(m)}(r)\right] B(r)\left[\begin{array}{c}
r^{3} b S_{n} \\
\frac{1}{b S_{n}}
\end{array}\right] \tilde{\phi}_{1}^{(n)}(r-1) d r \\
W_{m n} & =\int_{1}^{a}\left[\psi_{1}^{(m)}(r), \psi_{2}^{(m)}(r)\right] B(r)\left[\begin{array}{c}
r^{3} b P_{n} \\
\frac{1}{b P_{n}}
\end{array}\right] \hat{\phi}_{1}^{(n)}(r-1) d r
\end{aligned}
$$

and then making use of the biorthogonality condition, we get

$$
0=-2 \lambda_{m} F_{m} \sin \left(\lambda_{m} \theta_{1}\right) P_{m}^{*}+\sum_{-\infty}^{\infty} \lambda_{n} F_{n} R_{m n} \sin \left(\lambda_{n} \theta_{1}\right)-\sum_{-\infty}^{\infty}\left[C_{n} V_{m n}+D_{n} W_{m n}\right]
$$


From Eqs. (4.66), (4.70), and (4.79) we have the following infinite system of equations to be solved for the coefficients $C_{n}, D_{n}$, and $F_{n}$ :

$$
\left\{\begin{array}{l}
0=Q_{m}-k_{m} C_{m}+\sum_{-\infty}^{\infty} F_{n} U_{m n} \cos \left(\lambda_{n} \theta_{1}\right), \\
0=\widehat{Q}_{m}-\hat{k}_{m} D_{m}+\sum_{-\infty}^{\infty} F_{n} \widehat{U}_{m n} \cos \left(\lambda_{n} \theta_{1}\right), \\
0=-2 \lambda_{m} F_{m} \sin \left(\lambda_{m} \theta_{1}\right) P_{m}^{*}+\sum_{-\infty}^{\infty} \lambda_{n} F_{n} R_{m n} \sin \left(\lambda_{n} \theta_{1}\right)-\sum_{-\infty}^{\infty}\left[C_{n} V_{m n}+D_{n} W_{m n}\right] .
\end{array}\right.
$$

To rewrite the infinite system of equations (4.80) in matrix form, let

$$
\begin{aligned}
K & =2 \operatorname{diag}_{m}\left(\lambda_{m} P_{m}^{*} \sin \lambda_{m} \theta_{1}\right) \\
M & =\operatorname{diag}_{m}\left(\cos \lambda_{m} \theta_{1}\right) \\
H & =\operatorname{diag}_{m}\left(\lambda_{m} \sin \lambda_{m} \theta_{1}\right) \\
E & =\operatorname{diag}_{m}\left(\frac{1}{k_{m}}\right) \\
G & =\operatorname{diag}_{m}\left(\frac{1}{\hat{k}_{m}}\right)
\end{aligned}
$$

Then the first equation in (4.80) in matrix form becomes

$$
E \mathbf{Q}+(E U M) \mathbf{F}=\mathbf{C} .
$$

Similarly the second equation in $(4.80)$ becomes

$$
G \widehat{\mathbf{Q}}+(G \widehat{U} M) \mathbf{F}=\mathbf{D}
$$

and the third equation in (4.80) becomes

$$
(R H-K) \mathbf{F}-V \mathbf{C}-W \mathbf{D}=\mathbf{0} .
$$

Substituting Eqs. (4.82)-(4.83) into (4.84) we get

$$
\mathbf{O}=(R H-K) \mathbf{F}-V(E \mathbf{Q}+E U M \mathbf{F})-W(G \widehat{\mathbf{Q}}+G \widehat{U} M \mathbf{F}) .
$$

This gives

$$
V E \mathbf{Q}+W G \widehat{\mathbf{Q}}=(R H-K-V E U M-W G \widehat{U} M) \mathbf{F}
$$

Therefore we have

$$
\left\{\begin{array}{l}
\mathbf{F}=(R H-K-V E U M-W G \widehat{U} M)^{-1}(V E \mathbf{Q}+W G \widehat{\mathbf{Q}}), \\
\mathbf{C}=E \mathbf{Q}+E U M \mathbf{F}, \\
\mathbf{D}=G \widehat{\mathbf{Q}}+G \widehat{U} M \mathbf{F},
\end{array}\right.
$$

provided that the inverse of $S$ exists. Here

$$
S=R H-K-V E U M-W G \widehat{U} M .
$$


We note here that the $C_{i}^{\prime}$ s and $D_{i}^{\prime}$ s are given explicitly in terms of the $F_{i}^{\prime}$ s. System (4.85) represents an infinite system of equations for the components of the vector $\mathbf{F}$; this system is to be solved by truncation.

5. Numerical results and discussion. The first example we consider is flow in the region where $a=3, b=1$, and $\theta_{1}=\frac{\pi}{2}$ (see Fig. 1). The two subregions (I) and (II) shown in Fig. 1 are thus given respectively by

$$
\begin{aligned}
\mathcal{V}_{\mathrm{I}} & =\{X, Y:-\infty<X \leq 0,0 \leq Y \leq 2\}, \\
\mathcal{V}_{\mathrm{II}} & =\left\{r, \theta: 1 \leq r \leq 3,0 \leq \theta \leq \frac{\pi}{2}\right\}
\end{aligned}
$$

The complex eigenvalues given in (2.15), (2.16), and (2.27) were found to be numerically accurate to eight decimal places using a subroutine from the IMSL Library based on Muller's method with deflation (see Tables 1-2). A discussion of these eigenvalues is given in [13].

Equations (4.85) form an infinite system of equations to be solved for the coefficients $C_{n}, D_{n}$, and $F_{n}, n= \pm 1, \pm 2, \ldots$ To solve this system of equations we must truncate the infinite sums appearing in (3.56)-(3.59) to finite ones. We do this by replacing the lower and upper limits of summations by $-N$ and $N$, respectively, for region (I) and by $-\frac{1}{2} N$ and $\frac{1}{2} N$ for region (II). The truncated systems are then solved using a subroutine from the LINPACK Library based on Gaussian elimination with partial pivoting to compute the LU factorization of the complex matrix and then solve it. The coefficients of the truncated system that are given as integrals are integrated numerically using a subroutine from the IMSL Library based on the Gaussian Method.

The convergence of the solution of the truncated equations was then checked numerically at twenty mesh points on the interface between subregions (I) and (II). Convergence is reached when $N$ is 24 where the two solutions $\Psi_{\mathrm{I}}, \Psi_{\text {II }}$ together with their first three

TABLE 1. Thirteen first-quadrant eigenvalues of (2.15)-(2.16) for $a=3$

\begin{tabular}{|l|r||l|r|}
\hline$n$ & Complex roots $P_{n}$ & $n$ & Complex roots $S_{n}$ \\
\hline 1 & $3.748838139+1.384339142 \mathrm{i}$ & 1 & $2.106196115+1.125364306 \mathrm{i}$ \\
2 & $6.949979486+1.676104942 \mathrm{i}$ & 2 & $5.356268699+1.551574373 \mathrm{i}$ \\
3 & $10.119258854+1.858383840 \mathrm{i}$ & 3 & $8.536682427+1.775543674 \mathrm{i}$ \\
4 & $13.277273632+1.991570820 \mathrm{i}$ & 4 & $11.699177613+1.929404497 \mathrm{i}$ \\
5 & $16.429870503+2.096625735 \mathrm{i}$ & 5 & $14.854059913+2.046852462 \mathrm{i}$ \\
6 & $19.579408260+2.183397559 \mathrm{i}$ & 6 & $18.004933008+2.141890794 \mathrm{i}$ \\
7 & $22.727035732+2.257320224 \mathrm{i}$ & 7 & $21.153413359+2.221722915 \mathrm{i}$ \\
8 & $25.873384150+2.321713979 \mathrm{i}$ & 8 & $24.300342062+2.290552287 \mathrm{i}$ \\
9 & $29.018831030+2.378757559 \mathrm{i}$ & 9 & $27.446202894+2.351048230 \mathrm{i}$ \\
10 & $32.163616854+2.429958324 \mathrm{i}$ & 10 & $30.591295098+2.405012568 \mathrm{i}$ \\
11 & $35.307902531+2.476402679 \mathrm{i}$ & 11 & $33.735814317+2.453719208 \mathrm{i}$ \\
12 & $38.451800005+2.518899597 \mathrm{i}$ & 12 & $36.879894173+2.498102205 \mathrm{i}$ \\
13 & $41.595389719+2.558067733 \mathrm{i}$ & 13 & $40.023629218+2.538866867 \mathrm{i}$ \\
\hline
\end{tabular}


TABLE 2. Twenty-four first-quadrant eigenvalues of (2.27) for $a=3$

\begin{tabular}{|l|c|}
\hline$n$ & Complex roots $\lambda_{n}$ \\
\hline 1 & $2.240320591+3.798537445 \mathrm{i}$ \\
2 & $2.703035903+6.803103401 \mathrm{i}$ \\
3 & $3.004675284+9.735417974 \mathrm{i}$ \\
4 & $3.230082264+12.640111351 \mathrm{i}$ \\
5 & $3.410391280+15.530837167 \mathrm{i}$ \\
6 & $3.560758485+18.413395097 \mathrm{i}$ \\
7 & $3.689757283+21.290717883 \mathrm{i}$ \\
8 & $3.802724835+24.164463867 \mathrm{i}$ \\
9 & $3.903214445+27.035647620 \mathrm{i}$ \\
10 & $3.993713042+29.904927509 \mathrm{i}$ \\
11 & $4.076030031+32.772750823 \mathrm{i}$ \\
12 & $4.151523367+35.639432846 \mathrm{i}$ \\
13 & $4.221238428+38.505202650 \mathrm{i}$ \\
14 & $4.285997232+41.370230941 \mathrm{i}$ \\
15 & $4.346457904+44.234647703 \mathrm{i}$ \\
16 & $4.403155568+47.098553770 \mathrm{i}$ \\
17 & $4.456531219+49.962028630 \mathrm{i}$ \\
18 & $4.506952594+52.825135853 \mathrm{i}$ \\
19 & $4.554729554+55.687926903 \mathrm{i}$ \\
20 & $4.600125628+58.550443935 \mathrm{i}$ \\
21 & $4.643366815+61.412721813 \mathrm{i}$ \\
22 & $4.684648390+64.274789640 \mathrm{i}$ \\
23 & $4.724140232+67.136671915 \mathrm{i}$ \\
24 & $4.761991047+69.998389411 \mathrm{i}$ \\
\hline
\end{tabular}

partial derivatives with respect to $X$ given in Eqs. (3.28)-(3.31) match very well along the interface (Tables 3 and 4). The solution remains unchanged if $N$ is larger than 24 .

Figure 2 (see p. 590) shows the velocity profile $u$ in the $X$-direction for a parabolic flow (i.e., flow between two fixed parallel plates) which is contributed by the $\Psi_{0}(Y)$ term of the solution $\Psi_{\mathrm{I}}(X, Y)$ in subregion (I) given in Eq. (2.10) or in the case of subregion (II) is contributed by the $\widehat{\Psi}_{0}(r)$ term shown in Fig. 5 (see p. 592) of the solution $\Psi_{1 I}(r, \theta)$ given in Eq. (2.25). Figure 3 (see p. 591) shows the velocity profile $u$ at different values of $X$ in subregion (I) that is contributed by the infinite sum term in Eq. (2.10) while Fig. 6 (see p. 592) shows the peripheral (azimuthal) velocity profile $v_{\theta}$ at different angles $\theta$ in subregion (II) that is contributed by the infinite sum term in (2.25); these infinite sum terms add a small perturbation to the velocity profile of the parabolic and circular flows respectively. Flow starts at $X=-\infty$ where the flow remains parabolic since the contribution of the infinite sum is negligible (see Fig. 3) until $X=-1$ where the effect of the bend starts to be felt; an increase in $X$ from -1 to 0 is accompanied by a steady increase in the tilt of the parabolic profile towards the lower edge $Y=0$, see Fig. 1 , and the increase reaches its maximum at the interface where the tilt is towards the shorter edge $r=1$. Figure 6 shows that in subregion (II) and close to $\theta=0$ we have negligible 
TABle 3. Convergence of the Papkovitch-Fadle series for $N=24$, $a=3$, and $\theta_{1}=\frac{\pi}{4}$

\begin{tabular}{|c|c|c||c|c|}
\hline$r=Y+1$ & $\Psi_{\mathrm{I}}(0, Y ; 12)$ & $\Psi_{\mathrm{II}}\left(r, \frac{\pi}{4} ; 24\right)$ & $\frac{\partial \Psi_{\mathrm{I}}}{\partial X}(0, Y ; 12)$ & $\frac{\partial \Psi_{\mathrm{II}}}{\partial X}\left(r, \frac{\pi}{4} ; 24\right)$ \\
\hline 1.0 & $-1.19 \times 10^{-18}$ & $4.97 \times 10^{-18}$ & $-1.86 \times 10^{-17}$ & $1.61 \times 10^{-17}$ \\
1.1 & 0.009005 & 0.009015 & 0.009440 & 0.015947 \\
1.2 & 0.033418 & 0.033411 & 0.036029 & 0.034911 \\
1.3 & 0.071125 & 0.071129 & 0.061953 & 0.062951 \\
1.4 & 0.119558 & 0.119558 & 0.089948 & 0.088998 \\
1.5 & 0.176679 & 0.176680 & 0.114464 & 0.115384 \\
1.6 & 0.240459 & 0.240462 & 0.137870 & 0.136871 \\
1.7 & 0.309240 & 0.309238 & 0.154858 & 0.156011 \\
1.8 & 0.381263 & 0.381264 & 0.169676 & 0.168209 \\
1.9 & 0.455115 & 0.455114 & 0.175922 & 0.178132 \\
2.0 & 0.529160 & 0.529158 & 0.181445 & 0.177563 \\
2.1 & 0.602304 & 0.602303 & 0.172822 & 0.179973 \\
2.2 & 0.672666 & 0.672665 & 0.173646 & 0.161786 \\
2.3 & 0.739939 & 0.739938 & 0.146791 & 0.160651 \\
2.4 & 0.801651 & 0.801652 & 0.135240 & 0.134815 \\
2.5 & 0.857265 & 0.857266 & 0.132789 & 0.091918 \\
2.6 & 0.907894 & 0.907894 & 0.059537 & 0.113754 \\
2.7 & 0.949616 & 0.949617 & -0.019140 & 0.138451 \\
2.8 & 0.978361 & 0.978360 & -0.027293 & -0.093853 \\
2.9 & 0.994631 & 0.994633 & -0.005050 & 0.027613 \\
3.0 & 1.000000 & 1.000000 & $-1.05 \times 10^{-11}$ & $-7.46 \times 10^{-18}$ \\
\hline
\end{tabular}

contribution from the infinite sum term; so we almost have a concentric flow; as the angle increases we have an increase in the tilt of the profile towards the shorter circular edge $r=1$ compared with that of the concentric flow profile, and the tilt reaches its maximum when $\theta=80^{\circ}$; so as flow moves from subregion (II) to (I), i.e., close to the interface, there is a maximum tilt of the circular flow profile towards the shorter circular edge $r=1$ and this tilt decreases steadily as the flow in the bend moves away from the interface and the tilt reaches its minimum at $\theta=0$. Figures 4 and 7 (see pp. 591 and 593) show the contribution of the infinite sum terms on the velocity profile along the center-line of the channel; they clearly show that in region (I) and away from the interface the velocity is the same as of a parabolic flow. Then as we move along the center-line towards the interface there is a steady decrease in the velocity while in region (II) and very close to the interface we have a sharp and significant increase in the velocity (compared with the velocity of a circular flow). As we move further away from the bend and also close to $\theta=0$ we have negligible change in the velocity. The velocity components for subregions (I) and (II) are given by Eqs. (2.4) and (2.5), respectively. 
TABLE 4. Convergence of the Papkovitch-Fadle series for $N=24$, $a=3$, and $\theta_{1}=\frac{\pi}{4}$

\begin{tabular}{|c|c|c||c|c|}
\hline$r=Y+1$ & $\frac{\partial^{2} \Psi_{I}}{\partial X^{2}}(0, Y ; 12)$ & $\frac{\partial^{2} \Psi_{\mathrm{II}}}{\partial X^{2}}\left(r, \frac{\pi}{4} ; 24\right)$ & $\frac{\partial^{3} \Psi_{\mathrm{I}}}{\partial X^{3}}(0, Y ; 12)$ & $\frac{\partial^{3} \Psi_{\mathrm{I}}}{\partial X^{3}}\left(r, \frac{\pi}{4} ; 24\right)$ \\
\hline 1.0 & $-3.5 \times 10^{-15}$ & $3.7 \times 10^{-13}$ & $-1.8 \times 10^{-13}$ & $-4.0 \times 10^{-12}$ \\
1.1 & 0.120635 & 0.135068 & 0.990890 & 0.984468 \\
1.2 & 0.147559 & 0.139497 & 0.607406 & 0.606532 \\
1.3 & 0.198386 & 0.203048 & 0.743839 & 0.748482 \\
1.4 & 0.208850 & 0.207347 & 0.525435 & 0.530322 \\
1.5 & 0.230399 & 0.228013 & 0.397608 & 0.397706 \\
1.6 & 0.217373 & 0.222288 & 0.517091 & 0.507680 \\
1.7 & 0.229147 & 0.225638 & 0.280920 & 0.269147 \\
1.8 & 0.205211 & 0.205757 & 0.278058 & 0.285448 \\
1.9 & 0.211240 & 0.212460 & 0.267604 & 0.284524 \\
2.0 & 0.172618 & 0.171393 & 0.188027 & 0.169303 \\
2.1 & 0.197414 & 0.197475 & 0.178111 & 0.168899 \\
2.2 & 0.121621 & 0.122515 & 0.154856 & 0.191091 \\
2.3 & 0.170021 & 0.169100 & 0.124467 & 0.093415 \\
2.4 & 0.131333 & 0.131339 & 0.122110 & 0.115282 \\
2.5 & 0.007766 & 0.008791 & 0.105055 & 0.149799 \\
2.6 & 0.108523 & 0.107302 & 0.098928 & 0.053639 \\
2.7 & 0.209794 & 0.210550 & 0.111104 & 0.116905 \\
2.8 & 0.131792 & 0.130942 & 0.082792 & 0.110277 \\
2.9 & 0.027644 & 0.030368 & 0.162720 & 0.155006 \\
3.0 & $-5.7 \times 10^{-12}$ & $-6.4 \times 10^{-15}$ & $-1.4 \times 10^{-10}$ & $8.2 \times 10^{-13}$ \\
\hline & & & & \\
\hline
\end{tabular}

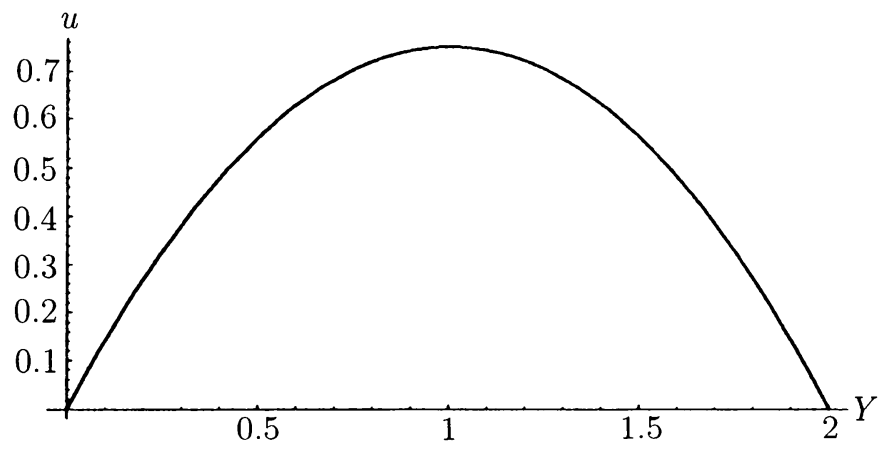

FIG. 2. Velocity profile $u$ for a parabolic flow contributed by the $\Psi_{0}(Y)$ terms given in (2.10) for subregion (I) given in (5.86) 


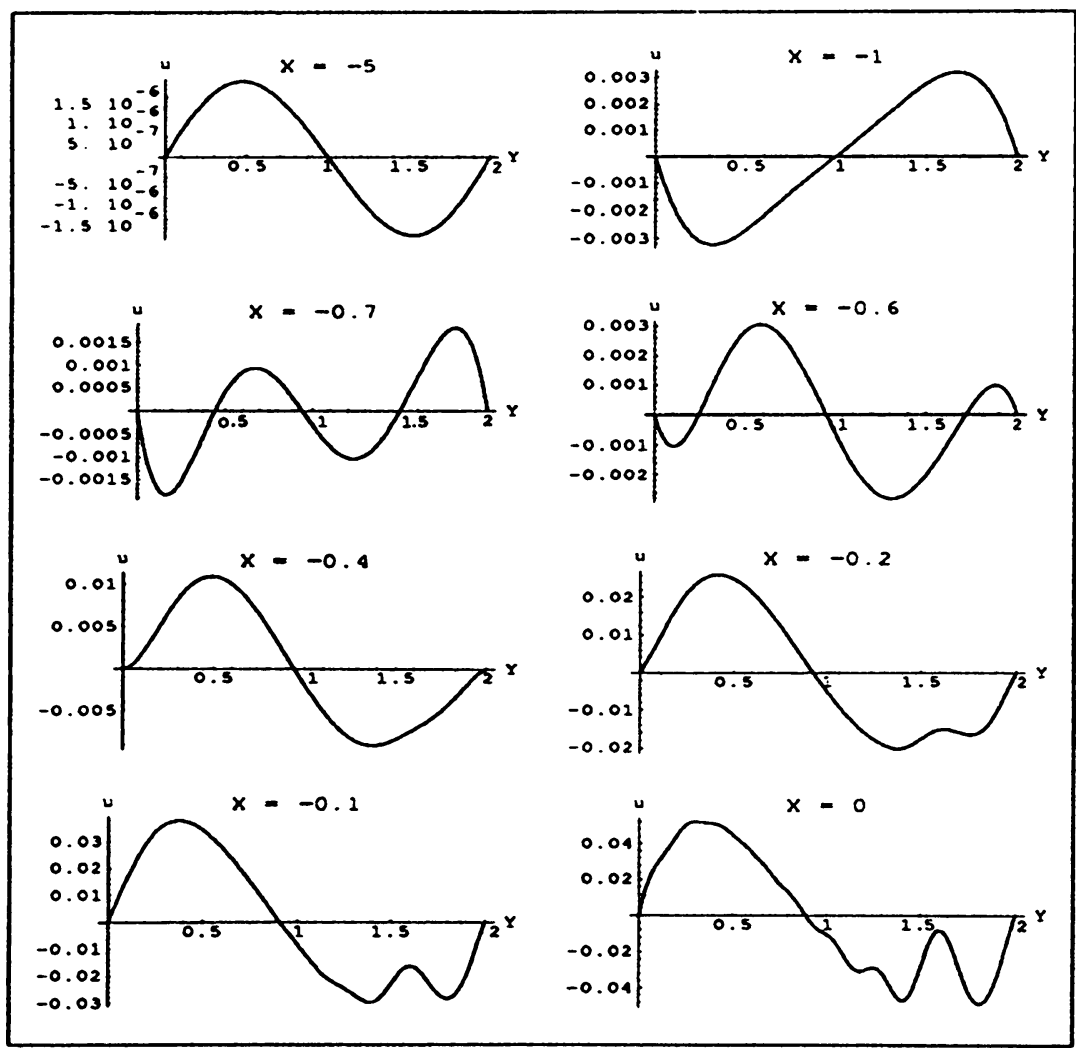

Fig. 3. Contribution of the infinite series term in (2.10) on the velocity profile $u$ given in (2.4), for the flow in subregion (I) given in (5.86), at different values of $X$

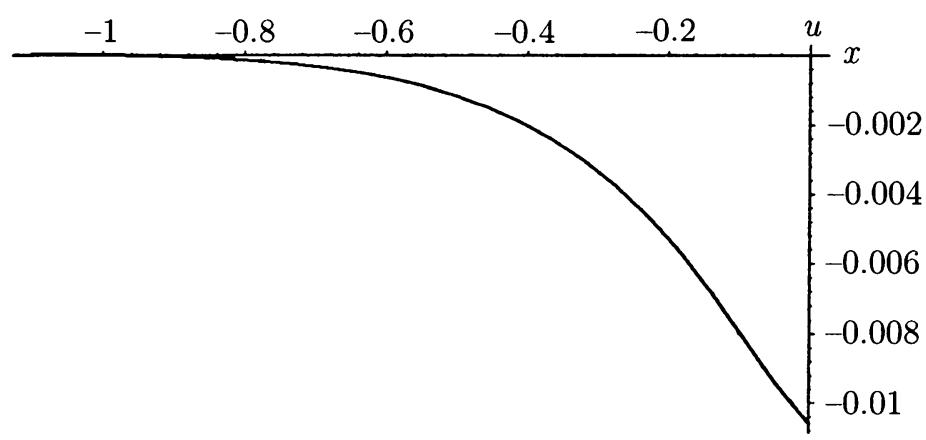

FIG. 4. Contribution of the infinite series term in (2.10) on the velocity profile $u$ given in (2.4), along the center-line $Y=1$ for the flow in subregion (I) given in (5.86) 


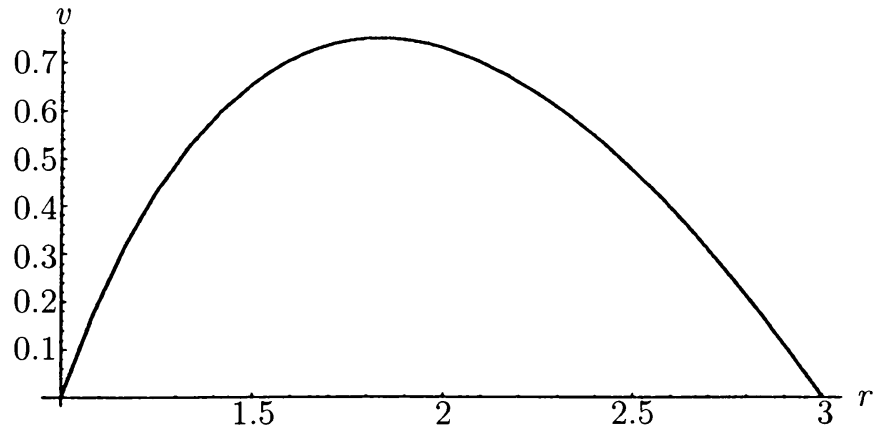

FIG. 5. Azimuthal velocity profile $v_{\theta}$ for a circular flow contributed by the $\widehat{\Psi}_{0}(r)$ term given in (2.25) for subregion (II) given in (5.87)

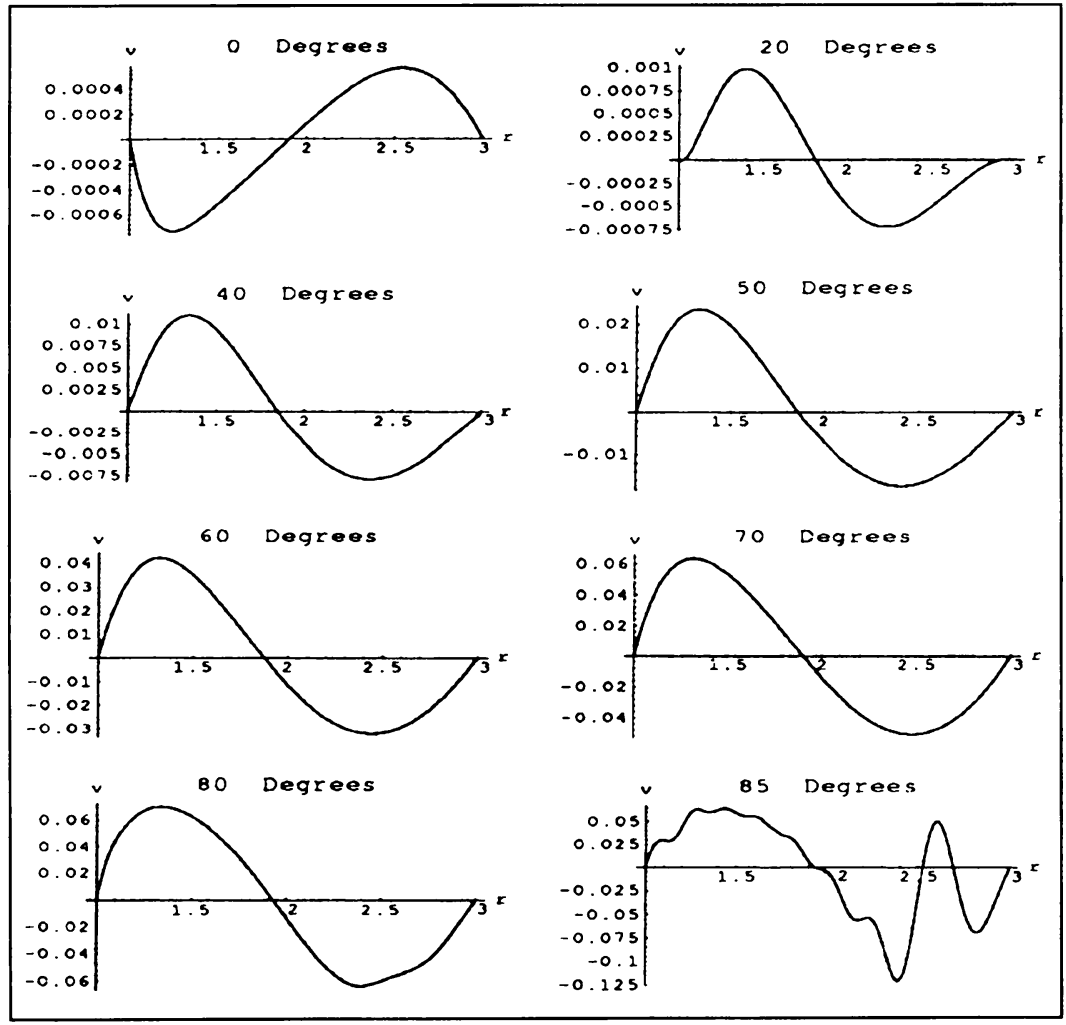

FIG. 6. Contribution of the infinite series term in (2.25) on the azimuthal velocity profile $v_{\theta}$, given in $(2.5)$, for the flow in subregion (II) given in (5.87), at different angles $\theta$ 


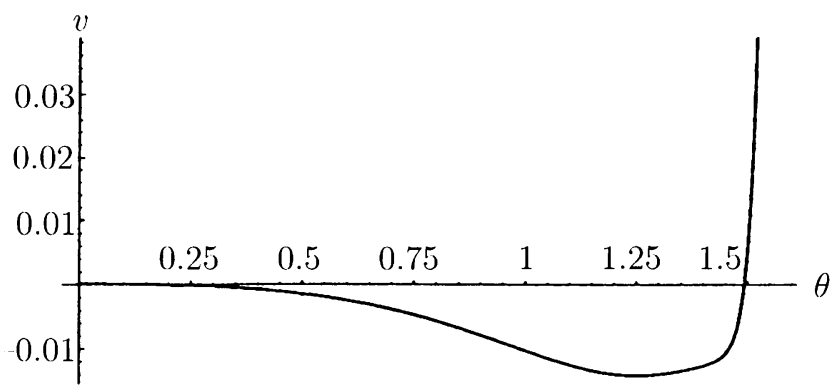

FIG. 7. Contribution of the infinite series term in (2.25) on the azimuthal velocity profile $v_{\theta}$, given in (2.5), along the center-line $r=2$ of the flow in subregion (II) given in (5.87)

The pressure $P$ and the velocity components in both regions (I) and (II) are related by the following equations:

$$
\begin{aligned}
& \frac{\partial P_{\mathrm{I}}}{\partial X}=\nabla^{2} u \\
& \frac{\partial P_{\mathrm{I}}}{\partial X}=\nabla^{2} v
\end{aligned}
$$

and

$$
\begin{aligned}
& \frac{\partial P_{\mathrm{II}}}{\partial r}=\nabla^{2} v_{r}-\frac{1}{r^{2}} v_{r}-\frac{2}{r^{2}} \frac{\partial v_{\theta}}{\partial \theta} \\
& \frac{\partial P_{\mathrm{II}}}{\partial \theta}=r\left(\nabla^{2} v_{\theta}+\frac{2}{r^{2}} \frac{\partial v_{r}}{\partial \theta}-\frac{v_{\theta}}{r^{2}}\right) .
\end{aligned}
$$

Substituting Eq. (2.10) into (2.4) we can compute the velocity components. Then using (5.88)-(5.89) leads, for subregion (I), to

$$
\begin{aligned}
& \frac{\partial P_{\mathrm{I}}}{\partial X}=\frac{-3}{2}+2 \sum_{-\infty}^{\infty} C_{n} S_{n} e^{S_{n} X} \cos S_{n} \sin S_{n}(Y-1)+D_{n} P_{n} e^{P_{n} X} \sin P_{n} \cos P_{n}(Y-1) \\
& \frac{\partial P_{\mathrm{I}}}{\partial Y}=2 \sum_{-\infty}^{\infty} C_{n} S_{n} e^{S_{n} X} \cos S_{n} \cos S_{n}(Y-1)-D_{n} P_{n} e^{P_{n} X} \sin P_{n} \sin P_{n}(Y-1) .
\end{aligned}
$$

Integrating Eq. (5.92), the pressure (up to an additive constant) is given by

$$
P_{\mathrm{I}}=-\frac{3}{2} X+2 \sum_{-\infty}^{\infty}\left\{C_{n} e^{S_{n} X} \cos S_{n} \sin \left[S_{n}(Y-1)\right]+D_{n} e^{P_{n} X} \sin P_{n} \cos \left[P_{n}(Y-1)\right]\right\}
$$




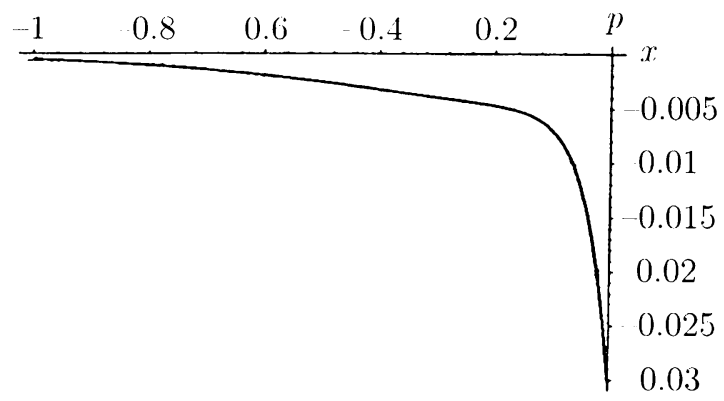

FIG. 8. Pressure at the center-line of the channel (i.e., where $r=2$ ) contributed by the infinite series term in (5.95) of subregion (I) given in $(5.86)$

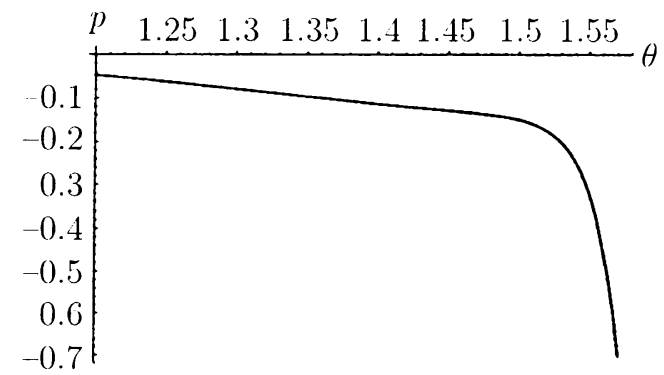

Fici. 9. Pressure at the center-line of the chamnel (i.e., where $r=$ 2) contributed by the nonconstant infinite series term in (5.97) of subregion (II) given in (5.87)

Substituting Eq. (2.25) into (2.5) we can compute the velocity components. Then using (5.90)-(5.91) leads, for subregion (II), to

$$
\begin{aligned}
& \frac{\partial P_{\mathrm{II}}}{\partial r}=-\sum_{-\infty}^{\infty} \lambda_{n} F_{n} \sin \left(\lambda_{n} \theta\right)\left[4\left(1-\lambda_{n}\right) c_{n} r^{-\lambda_{n}-1}+4\left(1+\lambda_{n}\right) r^{\lambda_{n}-1}\right], \\
& \frac{\partial P_{\mathrm{II}}}{\partial \theta}=\frac{64}{64-36 \log ^{2} 3}-\sum_{-\infty}^{\infty} F_{n} \cos \left(\lambda_{n} \theta\right)\left[4 \lambda_{n}\left(\lambda_{n}-1\right) c_{n} r^{-\lambda_{n}}+4 \lambda_{n}\left(\lambda_{n}+1\right) r^{\lambda_{n}}\right] .
\end{aligned}
$$

Integrating Eq. (5.94) and taking into account that the pressure is continuous across the interface, i.e., $P_{\mathrm{I}}=P_{\mathrm{II}}$, then

$$
\begin{aligned}
P_{\mathrm{II}}= & \frac{4}{64-36 \log ^{2} 3}\left(\theta-\frac{\pi}{2}\right)-\sum_{-\infty}^{\infty} F_{n} \sin \left(\lambda_{n} \theta\right)\left[4\left(\lambda_{n}-1\right) c_{n} r^{-\lambda_{n}}+4\left(1+\lambda_{n}\right) r^{\lambda_{n}}\right] \\
& +2 \sum_{-\infty}^{\infty} D_{n} \sin P_{n}+\sum_{-\infty}^{\infty} F_{n} \sin \left(\frac{\pi}{2} \lambda_{n}\right)\left[4\left(\lambda_{n}-1\right) c_{n} 2^{-\lambda_{n}}+4\left(1+\lambda_{n}\right) 2^{\lambda_{n}}\right] .
\end{aligned}
$$

Comparing Eqs. (5.93) and (5.95) we note that the pressure away from the interface is linear and the constant term appearing in (5.95) represents the pressure drop along the center-line of the channel. Contributions to the pressure along the center-line (i.e., where 
TABLE 5. Twenty-six first-quadrant eigenvalues of (2.27) for $a=2$

\begin{tabular}{|l|l|}
\hline$n$ & Complex roots $\lambda_{n}$ \\
\hline 1 & $3.370703254+6.054102744 \mathrm{i}$ \\
2 & $4.112343180+10.802931474 \mathrm{i}$ \\
3 & $4.593078513+15.444885446 \mathrm{i}$ \\
4 & $4.951563568+20.045543966 \mathrm{i}$ \\
5 & $5.238022153+24.625199349 \mathrm{i}$ \\
6 & $5.476764440+29.192525396 \mathrm{i}$ \\
7 & $5.681498446+33.751926784 \mathrm{i}$ \\
8 & $5.860740743+38.305901960 \mathrm{i}$ \\
9 & $6.020153718+42.855983209 \mathrm{i}$ \\
10 & $6.163696600+47.403166826 \mathrm{i}$ \\
11 & $6.294247926+51.948130806 \mathrm{i}$ \\
12 & $6.413966841+56.491353726 \mathrm{i}$ \\
13 & $6.524514778+61.033183727 \mathrm{i}$ \\
14 & $6.627197730+65.573880522 \mathrm{i}$ \\
15 & $6.723061006+70.113642063 \mathrm{i}$ \\
16 & $6.812954353+74.652622030 \mathrm{i}$ \\
17 & $6.897577913+79.190941676 \mathrm{i}$ \\
18 & $6.977515421+83.728698023 \mathrm{i}$ \\
19 & $7.053258673+88.265969687 \mathrm{i}$ \\
20 & $7.125225878+92.802821085 \mathrm{i}$ \\
21 & $7.193775648+97.339305529 \mathrm{i}$ \\
22 & $7.259217815+101.875467544 \mathrm{i}$ \\
23 & $7.321821895+106.411344626 \mathrm{i}$ \\
24 & $7.381823795+110.946968584 \mathrm{i}$ \\
25 & $7.439431179+115.482366591 \mathrm{i}$ \\
26 & $7.494827805+120.017562008 \mathrm{i}$ \\
\hline &
\end{tabular}

$r=2$ ) by the infinite sum terms appearing in (5.93), (5.95) are shown in Figures 8 and 9 , respectively, where close to the interface we have an abrupt and significant drop in the pressure, especially in region (II), i.e., around the bend.

The second example we consider is flow in a thinner channel where $a=2, b=2$, and $\theta_{1}=\frac{\pi}{4}$ (see Fig. 1). The two subregions (I) and (II) shown in Fig. 1 are thus given respectively by

$$
\begin{aligned}
\mathcal{V}_{\mathrm{I}} & =\{X, Y:-\infty<X \leq 0,0 \leq Y \leq 1\}, \\
\mathcal{V}_{\mathrm{II}} & =\left\{r, \theta: 1 \leq r \leq 2,0 \leq \theta \leq \frac{\pi}{4}\right\}
\end{aligned}
$$

In this case we decreased the width of the channel given in the previous example from 2 to 1 . Figures 10-15 (see pp. 596-598) show the behavior of the velocity profiles in regions (I) and (II); the analysis is very similar to the previous two examples and, except for this thinner channel, the effect of the bend starts to be felt closer to the interface than that of the wider channel; for instance, in subregion (I) the flow is almost parabolic 


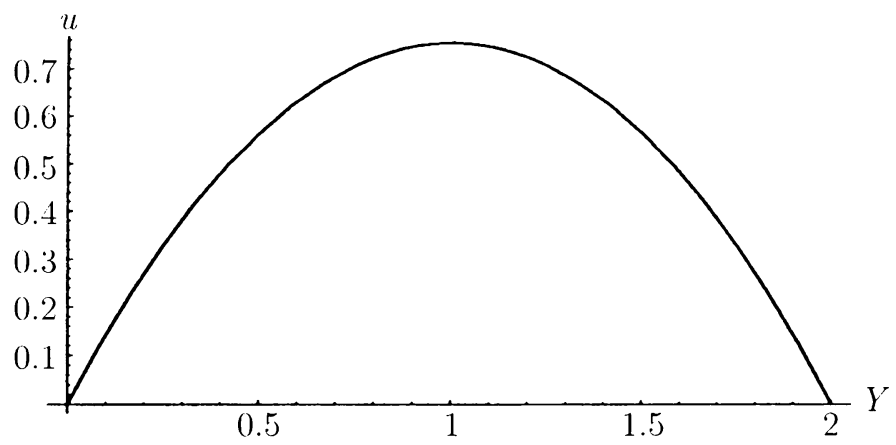

FI(i. 10. Velocity profile $u$ for a parabolic flow contributed by the $\Psi_{0}(Y)$ term given in $(2.10)$ for subregion (I) given in (5.96)

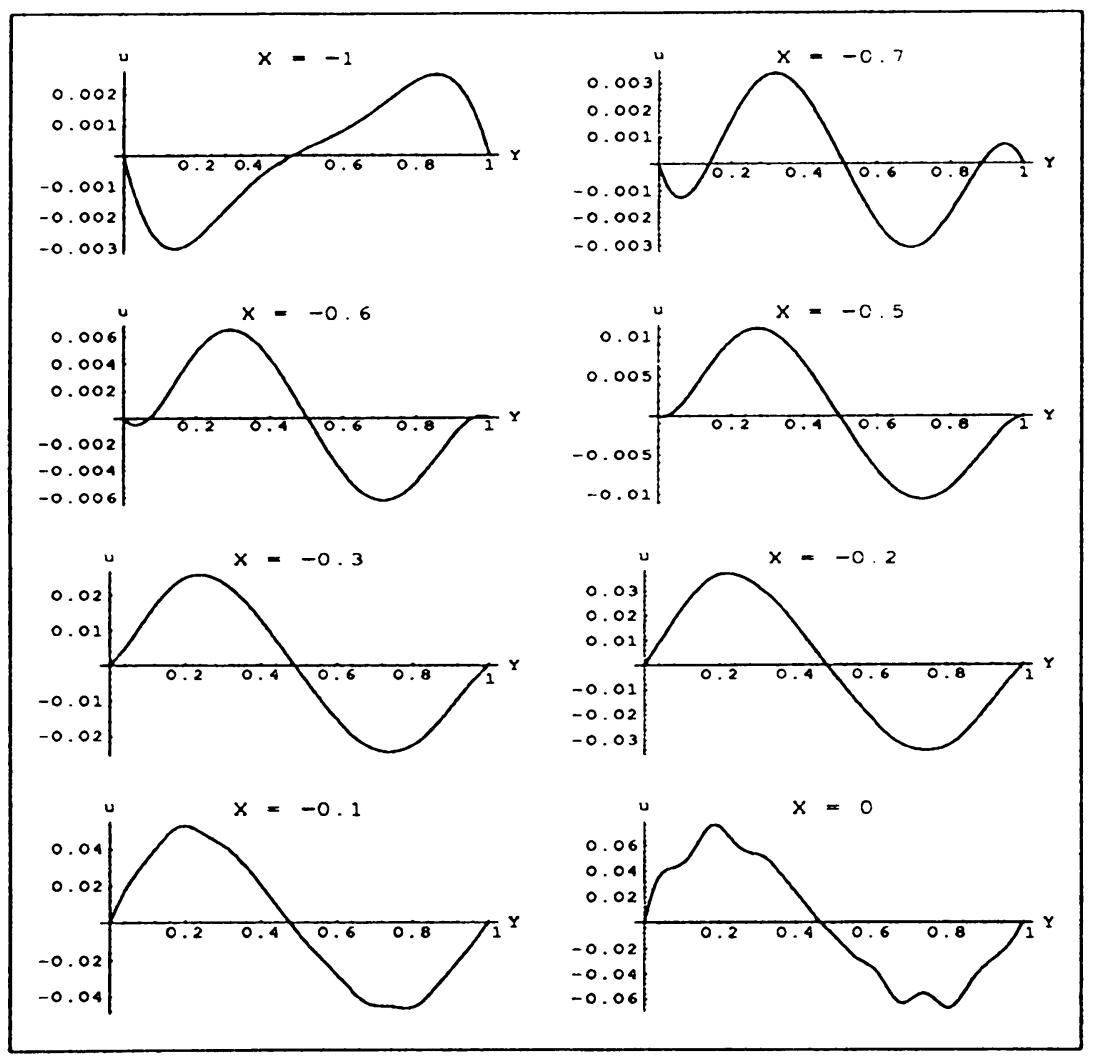

Fic: 11. Contribution of the infinite series term in (2.10) on the velocity profile $u$ given in (2.4), for the flow in subregion (I) given in (5.96), at different values of $X$ 


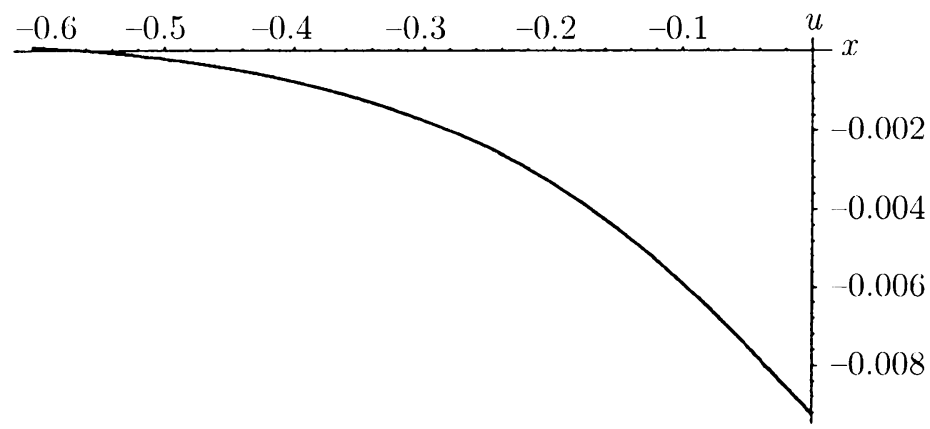

FIG. 12. Contribution of the infinite series term in (2.10) on the velocity profile $u$ given in (2.4), along the center-line $Y=\frac{1}{2}$ for the flow in subregion (I) given in (5.96)

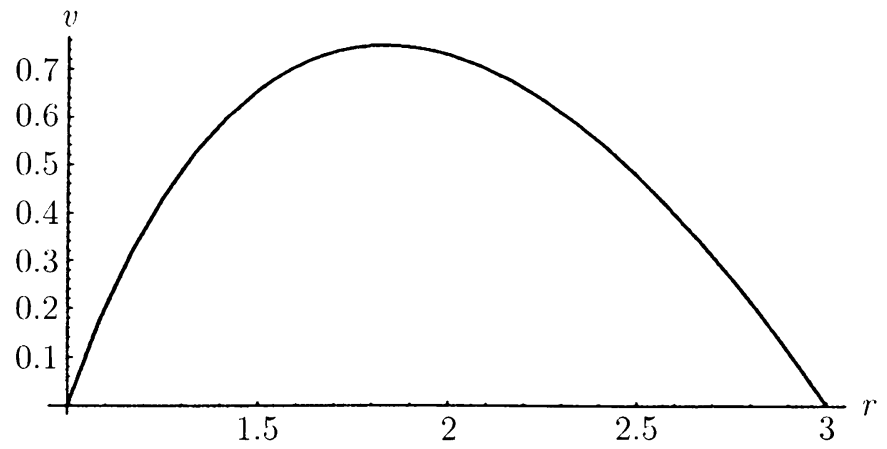

FIG. 13. Azimuthal velocity profile $v_{\theta}$ for a circular flow contributed by the $\widehat{\Psi}_{0}(r)$ term given in $(2.25)$ for subregion (II) given in (5.97)

until $X=-0.5$ where we start getting an increase in the tilt of the profile towards the lower edge; however, in the previous wider channel the effect started further away from the interface, namely when $X=-1$.

The convergence of the solution of the truncated equations was then checked numerically at twenty mesh points on the interface between subregions (I) and (II). As in example 1 the two solutions $\Psi_{I}, \Psi_{I I}$ together with their first three partial derivatives with respect to $X$ given in Eqs. (3.28)-(3.31) match very well along the interface. 


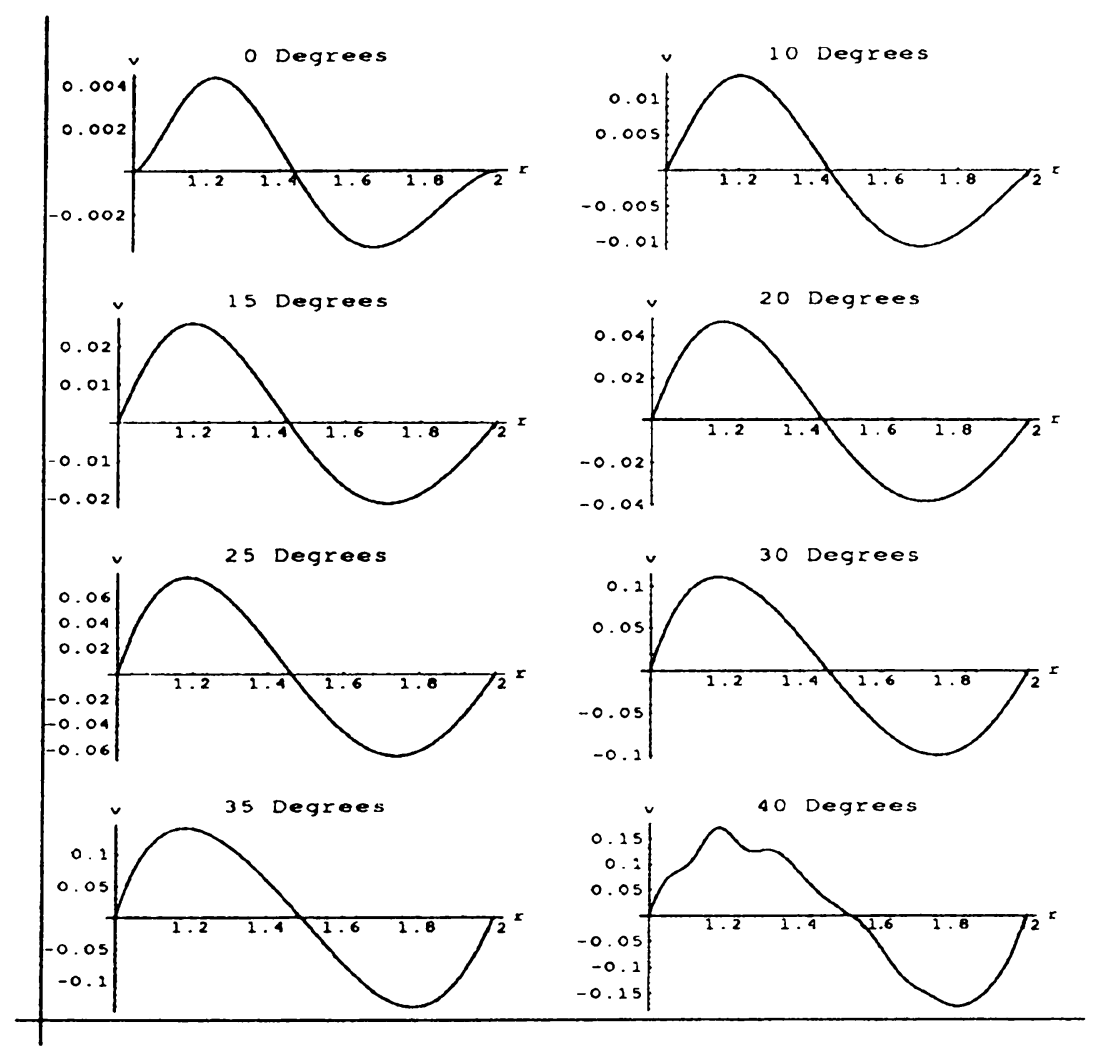

Fig. 14. Contribution of the infinite series term in (2.25) on the azimuthal velocity profile $v_{\theta}$, given in $(2.5)$, for the flow in subregion (II) given in $(5.97)$, at different angle $\theta$

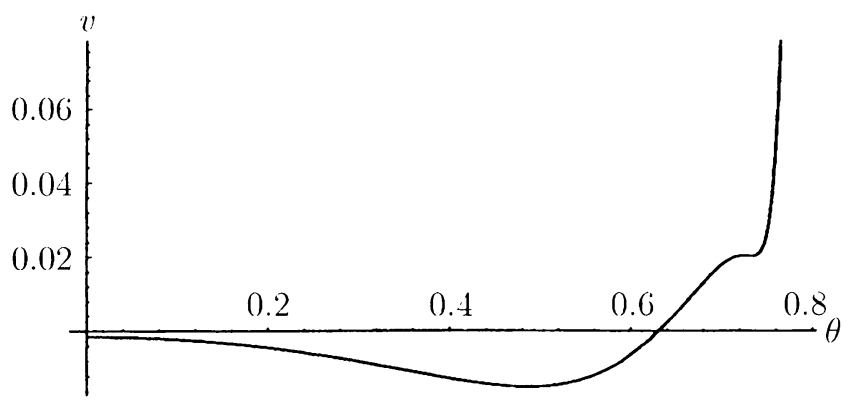

FIG. 15. Contribution of the infinite series term in (2.25) on the azimuthal velocity profile $v_{\theta}$, given in (2.5), along the center-line $r=\frac{3}{2}$ of the flow in subregion (II) given in (5.97) 
6. Conclusions. In this paper, the matched eigenfunction expansion method is used for solving Stokes flow around a channel bend. The flow region is decomposed into a rectangular and a cylindrical subregion. In each subregion the stream function is represented by means of an expansion of Papkovich-Fadle eigenfunctions. The coefficients in these expansions are determined by requiring weak $C^{3}$ continuity of the stream function across subregion interfaces. Advantage is taken of the biorthogonality conditions in both cylindrical and rectangular coordinates.

This example illustrates the matching method for two different coordinate systems where the biorthogonality conditions in both systems are utilized. The method can be generalized to other flow regions that can be partitioned into domains defined in different coordinate systems, as long as the biorthogonality conditions in both coordinates are given.

One advantage of the present method is that the flow regions can be treated without limiting to finite domains, as in finite-element or finite-difference methods (see, for example, [12]). In addition, due to the rapid convergence, only a few terms of the eigenfunction expansion are required to obtain accurate results. The computational time is much less than that needed for the direct numerical integration of the biharmonic equation.

\section{REFERENCES}

[1] A. J. Chorin and J. E. Marsden, A Mathematical Introduction to Fluid Mechanics, Springer-Verlag, 1990

[2] Z. Dagan, S. Weinbaum, and R. Pfeffer, An infinite-series solution for the creeping motion through an orifice of finite length, J. Fluid Mech. 115, $505-523$ (1982)

[3] J. Happel and H. Brenner, Low Reynolds Number Hydrodynamics, Prentice-Hall, NJ, 1965

[4] D. D. Joseph, Slow motion and viscometric motion; Stability and bifurcation of the rest state of a simple fluid, Arch. Rational Mech. Anal. 56, 99-156 (1974)

[5] D. D. Joseph, The convergence of biorthogonal series for biharmonic and Stokes flow edge problems, Part I, SIAM J. Appl. Mech. 33, 337347 (1977)

[6] D. D. Joseph, A new separation of variables theory for problems of Stokes flow and elasticity, In Trends in Applications of Pure Mathematics to Mechanics, Vol. II (H. Zorski, Ed.), London, Pitman. 1979

[7] D. D. Joseph and R. L. Fosdick, The free surface on a liquid between cylinders rotating at different speeds, Part I, Arch. Rational Mech. Anal. 49, 321-380 (1973)

[8] D. D. Joseph and L. Sturges, The convergence of biorthogonal series for biharmonic and Stokes flow edge problems, Part II, SIAM J. Appl. Math. 34, 7-26 (1978)

[9] D. D. Joseph and L. Sturges, The free surface on a liquid filling a trench heated from its side, J. Fluid Mech. 69, 565 589 (1975)

[10] D. D. Joseph, L. Sturges, and W. H. Warner, Convergence of biorthogonal series of biharmonic eigenfunctions by the method of Titchmarsh, Arch. Rational Mlech. Anal. 78, 223-274 (1982)

[11] A. Karageorghis and T. N. Phillips, Spectral collocation methods for Stokes flow in contraction geometries and unbounded domains, .J. Comput. Phys. 80, 314-330 (1989)

[12] M. Kawaguti, Numerical study of the flow of a viscous fluid in a curved channel, Physics of Fluids, vol. 12, 1969, pp. II-101 to 104

[13] S. A. Khuri, Biorthogonal series solution of Stokes flow problems in sectorial regions, SIAM J. Appl. Math. 56, No. 1, 19-39 (1996)

[14] W. E. Langlois. Slow Viscous Flow, Macmillan Company, NY. 1964

[15] C. H. Liu and D. D. Joseph, Stokes flow in wedge-shaped trenches, J. Fluid Mech. 42, 443-463 (1977)

[16] C. H. Liu and D. D. Joseph, Stokes flow in conical trenches, SIAM J. Appl. Math. 34, 286-296 (1978)

[17] T. N. Phillips, Singular matched eigenfunction expansions for Stokes flow around a corner, IMA Journal of Applied Mathematics 42, 13 26 (1989) 
[18] T. N. Phillips and A. R. Davies, On semi-infinite spectral elements of Poisson problems with reentrant boundary singularities, J. Computational Appl. Math. 21, 173188 (1988)

[19] T. N. Phillips and A. Karageorghis, Efficient direct methods for solving the spectral collocation equations for Stokes flow in rectangularly decomposable domains, SIAM J. Sci. Stat. Comput. 10, 89-103 (1989)

[20] T. N. Phillips and A. Karageorghis, A conforming spectral collocation strategy for Stokes flow through a channel contraction, Applied Numerical Nathematics 7, 329-345 (1991)

[21] L. C. Poteete, A matching technique for solving Stokes flow problems, Ph.D. Dissertation, Dept. Math., Michigan State Univ., 1993

[22] S. A. Trogdon and D. D. Joseph, Matched eigenfunction expansions for slow flow over a slot, J. Non-Newtonian Fluid Mech. 10, 185-213 (1982)

[23] C. Y. Wang, Exact solutions for the unsteady Navier-Stokes equations, Appl. Mech. Rev. 42, S269S282 (1989)

[24] J. Y. Yoo and D. D. Joseph, Stokes flow in a trench between concentric cylinders, SIAM J. Appl. Math. 34, 247285 (1978) 